University of Nebraska - Lincoln

DigitalCommons@University of Nebraska - Lincoln

3-2005

\title{
Dynamic Routing in Translucent WDM Optical Networks: The Intradomain Case
}

Xi Yang

IEEE

Byrav Ramamurthy

University of Nebraska-Lincoln, bramamurthy2@unl.edu

Follow this and additional works at: https://digitalcommons.unl.edu/csearticles

Part of the Computer Sciences Commons

Yang, Xi and Ramamurthy, Byrav, "Dynamic Routing in Translucent WDM Optical Networks: The Intradomain Case" (2005). CSE Journal Articles. 83.

https://digitalcommons.unl.edu/csearticles/83

This Article is brought to you for free and open access by the Computer Science and Engineering, Department of at DigitalCommons@University of Nebraska - Lincoln. It has been accepted for inclusion in CSE Journal Articles by an authorized administrator of DigitalCommons@University of Nebraska - Lincoln. 


\title{
Dynamic Routing in Translucent WDM Optical Networks: The Intradomain Case
}

\author{
Xi Yang, Member, IEEE, and Byrav Ramamurthy, Member, IEEE
}

\begin{abstract}
Translucent wavelength-division multiplexing optical networks use sparse placement of regenerators to overcome physical impairments and wavelength contention introduced by fully transparent networks, and achieve a performance close to fully opaque networks at a much less cost. In previous studies, we addressed the placement of regenerators based on static schemes, allowing for only a limited number of regenerators at fixed locations. This paper furthers those studies by proposing a dynamic resource allocation and dynamic routing scheme to operate translucent networks. This scheme is realized through dynamically sharing regeneration resources, including transmitters, receivers, and electronic interfaces, between regeneration and access functions under a multidomain hierarchical translucent network model. An intradomain routing algorithm, which takes into consideration optical-layer constraints as well as dynamic allocation of regeneration resources, is developed to address the problem of translucent dynamic routing in a single routing domain. Network performance in terms of blocking probability, resource utilization, and running times under different resource allocation and routing schemes is measured through simulation experiments.
\end{abstract}

Index Terms-Dynamic routing, physical impairments, regeneration, routing and wavelength assignment (RWA), signal quality constraints, translucent optical network, wavelength-division multiplexing (WDM).

\section{INTRODUCTION}

W AVELENGTH-DIVISION multiplexing (WDM) optical networks are providing huge bandwidth to keep up with the explosive growth of the Internet. Although most people are optimistic about the emergence of national-scale all-optical networks, in the current phase these networks must face the technical difficulties in overcoming the physical impairments introduced by long-haul fibers and cascading optical components such as erbium-doped fiber amplifiers (EDFAs) and optical cross-connects (OXCs) [1]. Physical impairments impose a fundamental limitation on the reach distance of signals in WDM optical networks. Wavelength continuity is another constraint that makes the design and operation of an all-optical network more complicated. A national-scale all-op-

Manuscript received July 17, 2003; revised October 18, 2004. This work was supported in part by the National Science Foundation under Grants ANI0074121 and EPS-0091900. An earlier version of this work appeared in Proceedings of IEEE International Conference on Communications (ICC), New York, NY, April 2002.

$\mathrm{X}$. Yang was with the Department of Computer Science and Engineering, University of Nebraska-Lincoln, Lincoln, NE 68588 USA. He is now with the University of Southern California Information Sciences Institute (USC/ISI) (e-mail: xyang@cse.unl.edu).

B. Ramamurthy is with the Department of Computer Science and Engineering, University of Nebraska-Lincoln, Lincoln NE 68588 USA (e-mail: byrav@cse.unl.edu).

Digital Object Identifier 10.1109/JLT.2004.841446 tical network will not appear in the near future before these technical obstacles are cleared.

An all-optical network is also called a transparent optical network [2]. The opposite of a transparent optical network is the opaque optical network [3]. An opaque optical network employs reamplifying, reshaping, and retiming (3R) regeneration at every intermediate node of a lightpath to clean up the signal and improve transmission quality. Currently, 3R regeneration can only be realized through optical-electronic-optical $(\mathrm{O} / \mathrm{E} / \mathrm{O})$ conversion. In the near future, $3 \mathrm{R}$ regeneration will remain the main method to extend the reach distance of signals, while $\mathrm{O} / \mathrm{E} / \mathrm{O}$ conversion will still be indispensable for a $3 \mathrm{R}$ regeneration capable optical network. However, the cost for an opaque network to regenerate every wavelength at every intermediate node is prohibitive. In previous studies [3]-[5], we proposed an alternate approach, the translucent network, which uses the sparse regeneration technique to allocate regeneration resources, i.e., transmitters $(\mathrm{E} / \mathrm{O})$, receivers $(\mathrm{O} / \mathrm{E})$, and electronic interfaces, in an efficient manner. In a translucent optical network, a signal is made to travel as long as possible before its quality degrades below a threshold or wavelength contention occurs. Because a signal is regenerated only if necessary, we need much less regeneration resources.

The concept of translucent network provides us a new perspective on the evolution of optical transport networks. The optical transport networks today are fully opaque. WDM technology only serves to improve link capacity. The reach distance of all-optical signals is limited to the span of a link, while the switching completely relies on the electronic layer, e.g., the SONET/SDH layer. With advances in optical switching technologies, the reach distance of all-optical signals has been extended to span more than one optical link without regeneration. The opaque networks today are evolving into the early translucent networks, which contain some isolated domains of transparency. A domain of transparency is a subnetwork that consists of boundary nodes and interior nodes. O/E/O processing of optical signals is only carried out at the boundary nodes. Inside the domain, optical signals are processed and transported transparently at the interior nodes. In this phase, the size of each domain of transparency is small with a few regeneration capable boundary nodes and more transparent interior nodes. The remaining network is still opaque. In [6], the author called those domains islands of transparency. When the evolution continues, we can envision that more domains will appear across the entire network and the size of a domain will become large. Many long-haul lightpaths will not need 3R regeneration anymore. The translucent network will consist of a number of adjacent large domains with only limited number of regeneration 


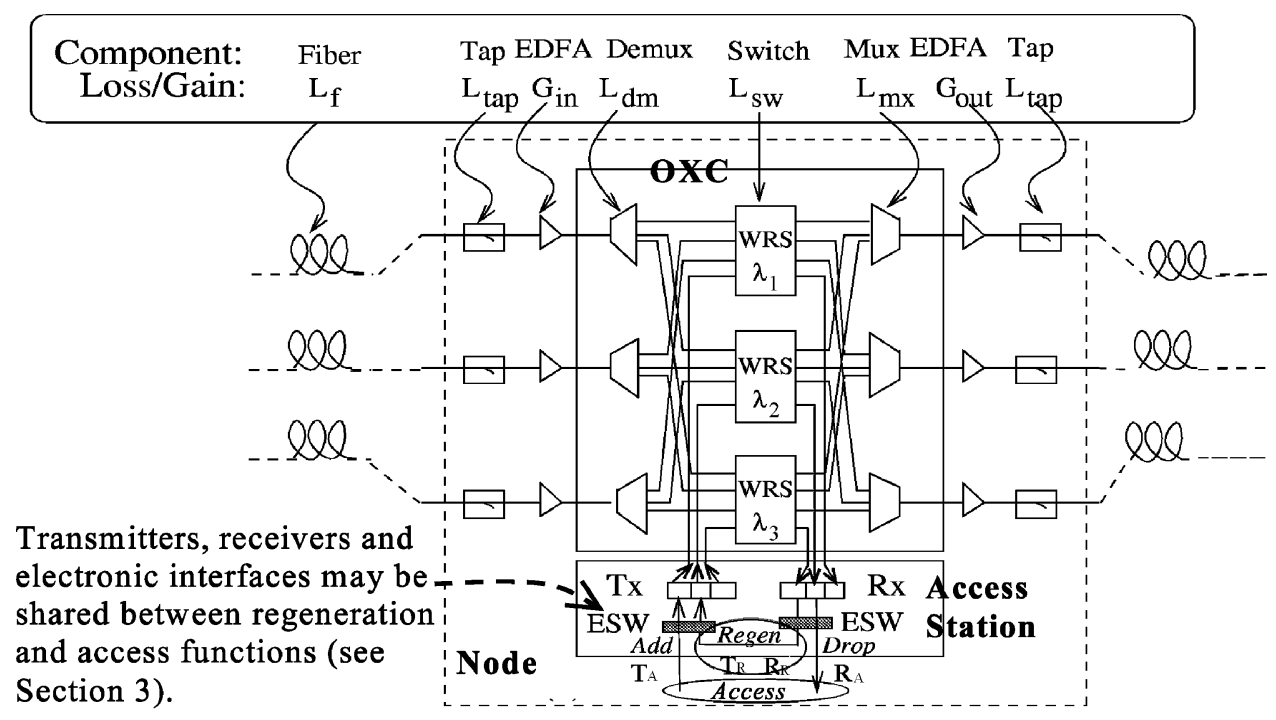

Fig. 1. Regeneration capable node model.

resources at sparse locations, accommodating regeneration for a small fraction of lightpaths. Eventually, the entire network will become fully transparent.

For a translucent network with isolated and small-sized domains of transparency, it is simple to regenerate every optical signal at the domain boundary while keeping the interior part of a domain fully transparent. The domains of transparency can be engineered only in optical domain so that physical impairments do not impose significant constraints on wavelength routing. In this case, both the network topology and the wavelength routing algorithm can be designed in a fixed or static manner. However, when the translucent network evolves into a number of adjacent large domains, the wavelength routing issue will be greatly complicated. First, optical-domain engineering can no longer effectively handle the physical impairments and wavelength contention for all possible lightpaths due to increased complexity. Second, the optical-layer constraints and available regeneration resources may change dynamically with the traffic so that a fixed or static routing algorithm becomes incapable of setting up those lightpaths efficiently. Third, in a large domain that contains a large number of nodes, it is wasteful to only regenerate signals at the boundary without utilizing the potential regeneration resources at those interior nodes. For these reasons, we propose a dynamic resource allocation and dynamic routing scheme, which is built up on the groundwork of the generalized multiprotocol label switching (GMPLS) [7] standards and drafts developed by the Internet Engineering Task Force (IETF).

The dynamic routing problem in a large network is often divided into separate intradomain routing and interdomain routing problems. The intradomain routing is supposed to address optical-layer constraints and allocation of regeneration resources in each domain and therefore is the focus of this study.

The rest of this paper is organized as follows. In Section II, we provide a review of related studies on translucent networks and dynamic routing issues. In Section III, we describe the dynamic resource allocation and dynamic routing scheme for operation of translucent networks. In Section IV, we formulate the problem of translucent dynamic routing for the intradomain case. In Section V, we develop an intradomain translucent dynamic routing algorithm. In Section VI, experimental results are presented. We conclude this paper in Section VII.

\section{REVIEW OF RELATED STUdiES}

\section{A. Our Previous Work}

In previous studies [3], [4], we proposed the translucent optical network architecture and examined its feasibility. A translucent optical network consists of a number of regeneration capable nodes. All other nodes are transparent nodes that process and transport optical signals only in optical domain. Fig. 1 is the model of a regeneration capable node in the translucent optical network. The node consists of an OXC and an access station. Regeneration resources include transmitters $\left(\mathrm{T}_{\mathrm{X}}\right)$, receivers $\left(\mathrm{R}_{\mathrm{X}}\right)$, and electronic interfaces in the access station. An electronic interface corresponds to an electronic port that supports a specific type of digital format at the electronic switches (ESWs). The type of digital format is represented by the combination of the digital rate and the frame format. It also refers to a combination of switching capability and capacity in IETF standards and drafts [7], e.g., switching capability: TDM and capacity: STM-16.

$T_{X}$ and $R_{X}$ are assigned into three sets: $T_{A}, R_{A}$, and $T_{R} R_{R}$. If a lightpath is at its source, it enters into the set $\mathrm{T}_{\mathrm{A}}$. If the lightpath is at its destination, it enters into the set $R_{A}$. If the lightpath is at an intermediate node, a pair of transmitter and receiver, called $T-R$ pair, in the set $T_{R} R_{R}$ can be allocated to regenerate this lightpath. In our previous studies on translucent network design, we had a fixed number of T-R pairs to be allocated and reserved for regeneration. Each T-R pair plus an electronic interface forms a regenerator. We used a technique called sparse regeneration to design the translucent network through sparse placement of those regenerators. Each regenerator was fixed at one node in a flat network using regenerator placement algorithms. The placement algorithms were carried out offline, 
i.e., at the network planning stage, based on the prediction of future traffic demands. We used the shortest path first (SPF) algorithm to calculate static routes for the predicted traffic demands. Experimental results showed that only $20 \%$ of regenerators are needed to achieve a performance close to an opaque network [5]. Although the sparse regeneration technique contributes to improved performance, the fixed routing and wavelength assignment algorithms are unaware of the regeneration resources (except for those at the nodes that a static route happens to traverse) and hence cannot efficiently utilize them.

\section{B. Other Relevant Work}

1) Sparse Regeneration: Other ongoing research also supports the idea of sparse regeneration in large-scale optical networks. As surveyed by [8], the ordinarily used $600 \mathrm{~km}$ reach distance for transparent optical signals is far from the requirement for today's Internet traffic. Even a reach distance of $3000 \mathrm{~km}$ can only satisfy $60 \%$ of the connections. However, current technologies have difficulty in extending the reach distance to more than $2000 \mathrm{~km}$ [9]. In [6], which is one of the earliest studies on domains of transparency, the authors suggested using "islands of transparency" to establish subconnections between regeneration sites. Similar to our previous studies [4], [5], the subconnections are established offline subject to physical constraints. In [10], the authors addressed the problem of maximizing the transparency advantage by following our research in [5]. In [11], the authors discussed the challenges for monitoring the performance of optical signals in optical networks. Note that our regeneration capable node model supports limited optical signal monitoring functionality using taps (see Fig. 1), which provides some information about physical impairments.

2) Dynamic Routing: This study is focused on wavelength routing in translucent optical networks. The routing issues in the Internet have been studied extensively, where a variety of interior gateway protocols, such as the open shortest path first (OSPF) and intermediate system-to-intermediate system (IS-IS) protocols, are used to route IP traffic inside each routing domain, while the border gateway protocol (BGP) is used to route traffic across multiple domains. This solution is both flexible and scalable because interdomain routing is separated from diverse and complex intradomain routing constraints, resource allocation schemes, and local policies. Inside a routing domain, each node maintains local adjacency states, and exchanges the adjacency states with other nodes to obtain global routing information. To meet the requirement for traffic engineering (TE), some performance metrics, such as packet loss and delay, can be incorporated into the modified Dijkstra's algorithm to obtain optimal routing solutions. Recent research efforts proposed to enhance such a scheme to route wavelengths in optical networks [12], [13]. Subject to the constraints on capacity, physical impairments, and wavelength continuity, the objective is minimizing the cost of desired routes. A wavelength routing algorithm that considers physical impairment constraints was presented in [14]. Most of those proposed routing algorithms use static or offline computation, which calculates routes prior to the arrival of route requests. Some dynamic or online routing algorithms were also proposed to satisfy the requests based on real-time network states [13]-[15]. Dynamic routing algorithms are more suitable in an environment where constraints change dynamically with traffic and costs are sensitive to resource utilization, which is exactly the case for the intradomain routing in a translucent optical network.

3) Standards: The proposal of dynamic routing in a translucent network is also supported by today's standardization efforts toward a unified control plane for next-generation optical networks. Routing is one aspect of the control plane functionality. Currently, the GMPLS-based control plane developed by IETF is the most recognized standardization effort and is very promising to become the standard for the control plane of optical transport networks [7], [16]. Using GMPLS signaling protocols, e.g., the latest TE enhancements of resource reservation protocol (RSVP-TE) [17] and label distribution protocol (CR-LDP) [18], optical transport network nodes can interoperate in the same way as label switched routers (LSRs) to establish lightpaths by means of label switched paths (LSPs) [19]. An additional benefit is that it supports a hierarchical routing and switching architecture. GMPLS supports extended label space and LSP hierarchy for TDM shims, optical wavelengths, wavebands and fibers. A lightpath spanning several optical links can be treated as a virtual link to achieve better scalability. The IP over Optical Working Group (IPO WG) of IETF is also working on enhancing the link management protocol (LMP) [20] to support management of TDM and WDM optical links [21], [22]. One of the IETF drafts studies the impact of physical impairments on optical-layer routing [23]. Some new issues raised from wavelength routing in optical transport networks are also discussed in the draft on routing extension for GMPLS [24]. The IETF standards and drafts build a solid groundwork for this paper. Our study of dynamic routing in translucent optical networks will comply with the architectures, protocols, and conventions of GMPLS.

\section{TRanslucent Dynamic Routing Scheme}

In this paper, we propose a dynamic resource allocation and dynamic routing scheme to perform wavelength routing in translucent optical networks. This scheme differs from those in previous studies in two aspects. First, the regeneration function can share transmitters, receivers, and electronic interfaces with the access function at any regeneration capable nodes. Normally the access function does not need to use all T-R pairs and electronic interfaces at every node. Some nodes must have spare T-R pairs and electronic interfaces remaining for the regeneration function. Each node with spare T-R pairs and electronic interfaces may become a regeneration node. Second, this scheme is aimed at the translucent network with multiple routing domains. It can regenerate signals not only at boundary nodes but also at interior regeneration capable nodes. A lightpath is allowed to traverse a boundary node or an interior regeneration capable node either through regeneration or through optical bypass.

\section{A. Network Model}

A translucent optical network can be modeled as an LSP routed network under the GMPLS architecture. We introduce a regeneration layer into the GMPLS LSP hierarchy. Note that 


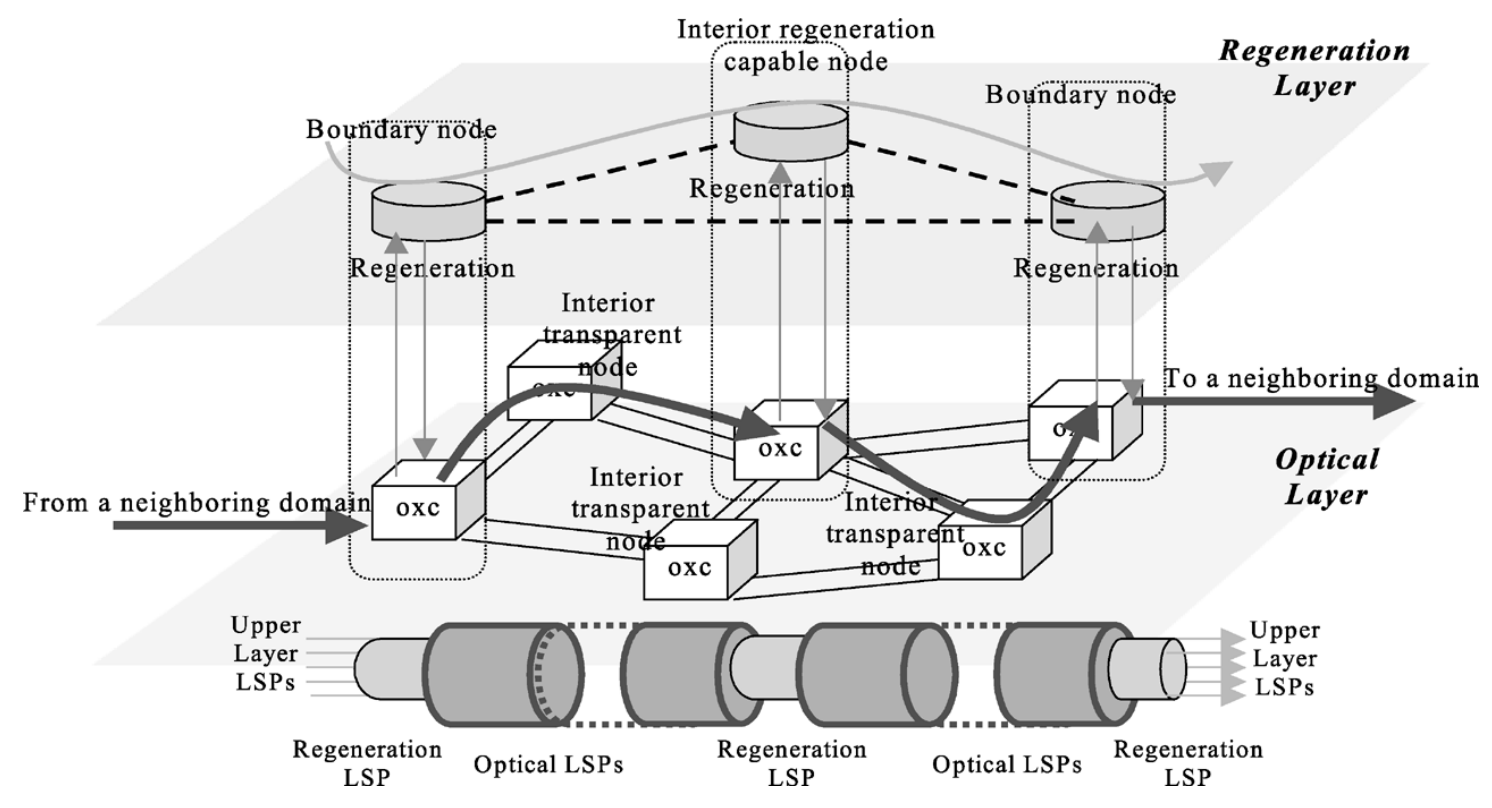

Fig. 2. Multidomain hierarchical translucent network model.

the terms regeneration layer, regeneration LPS, and regeneration link are only used to elaborate the idea of dynamic routing in translucent optical networks in this paper. We are not introducing new terms into GMPLS standards and drafts because the regeneration layer is abstract and can be incorporated as one of the existing electronic layers, e.g., the ITU-T G.709 digital path layer or the digital wrapper layer [25]. The most common example of a regeneration layer is the SONET/SDH layer, at which a regeneration LSP can be a gigabit Ethernet (GigE), STM-16, or STM-64 framed digital path. Fig. 2 illustrates the hierarchical translucent network model. The upper layer LSPs can be any electronic paths carrying IP, asynchronous transfer mode, frame relay, or Ethernet traffic. From the viewpoint of the upper layer, a regeneration LSP is simply a regeneration link. Each regeneration link is set up between a pair of electronic interfaces supporting the same type of digital format. A receiver at the upstream node and a transmitter at the downstream node are needed. One or more upper layer LSPs are aggregated into a wavelength-level end-to-end lightpath, which traverses multiple concatenated regeneration links or regeneration LSPs. Each regeneration LSP, e.g., a SONET/SDH framed digital path, is transported across multiple concatenated optical links. An optical link has at least one link property: the assigned wavelength index. We will use wavelength to represent wavelength index in the remainder of this paper.

In our model, a translucent network consists of multiple adjacent domains. Within each domain, regeneration resources are dynamically allocated not only at boundary nodes but also at interior regeneration capable nodes. In this regard, the domain does not strictly mean a domain of transparency any more. Both the boundary nodes and the interior regeneration capable nodes can be modeled using the regeneration capable node model (see Fig. 1), which allows for integration of the optical bypass, electronic access and regeneration functions. Other interior nodes are called interior transparent nodes, which only allow for optical bypass and electronic access, but do not allow for regen- eration. When an end-to-end lightpath is set up across multiple domains, it is divided into several route segments, each in one domain and having the same hierarchal LSP structure as shown in Fig. 2.

\section{B. Features}

Due to the sharing of transmitters, receivers, and electronic interfaces between the regeneration and access functions, the availability status of those resources will vary from time to time along with the change of traffic. To cope with the variation, the resources need to be managed in a dynamic manner. Their availability status needs to be advertised as global network states and be dynamically updated. It is impossible for a static or offline routing scheme to efficiently calculate routes in this situation. Only an online routing scheme using the dynamically updated status of regeneration resources is capable of automatically provisioning lightpaths with improved efficiency. The dynamic resource allocation and dynamic routing scheme is characterized by the following features. All these features are designed based on the current IETF standards and drafts on the routing and signaling aspects of GMPLS.

- Dynamic Resource Allocation: To share the regeneration resources between regeneration and access functions, the resource management module must support a series of operations that can dynamically allocate transmitters and receivers into the three sets: $T_{A}, R_{A}$, and $T_{R} R_{R}$, maintain their status, and map them to OXC ports. Electronic interfaces are similarly allocated and managed. Dynamic resource allocation and port mapping are regular signaling functions defined by GMPLS signaling protocols [26], e.g., CR-LDP and RSVP-TE.

- Link State Management: Under the GMPLS architecture, LMP provides a generic link management method independent of data channels. LMP is used to manage connectivity status and verify interfaces on both ends of 
a link [20]. Wavelength (index) is maintained as a link property on each optical link. Other link properties concerned with optical-layer constraints are also maintained. Because LMP supports virtual links across several physical links [27], a regeneration LSP that traverses multiple optical links is managed as a regeneration link. The type of digital format is maintained as a link property between two electronic interfaces on each such regeneration link.

- Advertisement and Discovery of Regeneration Resource States: The GMPLS extensions of link-state routing protocols with traffic engineering (TE) enhancements, such as OSPF-TE and IS-IS-TE, have been proposed to disseminate link states for TDM and WDM links [28], [29]. In particular, OSPF-TE uses link state advertisements (LSAs) to disseminate link states and TE parameters between network nodes. For interdomain routing in optical networks, the BGP/GMPLS solution has been proposed [30]. We also proposed an interdomain routing solution from an algorithmic perspective in [41]. All these enhancements can also be used for disseminating and updating states of regeneration and optical links in translucent optical networks.

- Hierarchical Routing Architecture: Fig. 2 also shows that the routing architecture in translucent networks has two layers. A lightpath may be established by concatenating multiple regeneration links, each corresponding to multiple underlying optical links. MPLS facilitates such a hierarchical routing architecture through LSP tunneling [27], which allows a virtual link to be formed by an LSP that tunnels concatenated underlying physical links. Therefore, dynamic wavelength routing in translucent optical networks can be carried out on the regeneration-layer network topology that consists of regeneration capable nodes and regeneration links. The regeneration links are dynamically established by routing regeneration LSPs on the optical-layer network topology that consists of optical cross-connects and optical links.

- Efficient Regeneration-Aware Routing Algorithm: Routing in a translucent optical network cannot just use simple objectives such as minimizing the number of optical links. New objectives, e.g., minimizing the number of regenerations, are required. Also, the constraints on physical impairments, wavelength continuity, and diversity result in complicated routing computation. Sparse regeneration in translucent optical networks adds to the complexity by introducing constraints on allocation of regeneration resources. Fortunately, under the GMPLS architecture, existing routing protocols with TE enhancements [24] are open to these new objectives and constraints arising in translucent optical networks.

\section{Problem Formulation: The Intradomain ROUTING CASE}

\section{A. Problem Statement}

According to the network model described in Section III, an end-to-end lightpath is established through one or more route segments, each corresponding to a local route in a single domain, thus resulting in two separate routing problems: the intradomain routing problem and the interdomain routing problem. This study is devoted to addressing the intradomain routing problem, in which we take into consideration optical-layer constraints as well as allocation of regeneration resources. By leaving the complex local routing constraints and computation to an intradomain routing algorithm, the interdomain algorithm only needs to concatenate those local routes into lightpaths at the domain boundary, and hence can be made simple and carried out "on-the-fly" [41].

In intradomain routing, we define four different roles for a local to serve in an end-to-end lightpath.

Rule A. The local route between a local source node $S$ and a local destination node $T$ serves as an intermediate segment in a lightpath that passes through both $S$ and $T$.

Rule B. The local route serves as the first segment in a lightpath that originates at $S$.

Rule C. The local route serves as the last segment in a lightpath that terminates at $T$.

Rule D. The local route serves as a complete lightpath that originates at $S$ and terminates at $T$.

Note that regeneration at the local source node $S$ is equivalent to electronic access at $S$ if the lightpath originates at $S$. Similarly, regeneration at the local destination node $T$ is equivalent to electronic access at $T$ if the lightpath terminates at $T$. Both regeneration and optical bypass can be used at an intermediary boundary node to concatenate two consecutive local routes. Next, we will describe what constraints should be used for the local routing computation and what objectives should be achieved when optical bypass and regeneration are carried out at the appropriate nodes on a local route under those constraints.

\section{B. Optical-Layer Constraints}

There are three categories of optical-layer constraints in optical wavelength routing: the constraints on physical impairments, the constraints on wavelength continuity, and the constraints on diversity (i.e., working and backup lightpaths must be routed through diverse paths to avoid any "single point of failure"). In this paper, we only address the first two categories of constraints.

1) Constraints on Physical Impairments: We consider the constraints on chromatic dispersion (CD), polarization mode dispersion (PMD), amplified spontaneous emission (ASE), and crosstalk due to mux/demultiplexers and cross-connects. Power loss can be compensated by appropriate placement of amplifiers so that we can simply ignore its impact, i.e., the signal power is assumed to be constant. We also ignore polarization dependent loss (PDL), nonlinear effects, and other impairments.

As a general rule of notation, we use the subscripts input and output to distinguish the measurements of physical impairments at the source and destination nodes of a local route. We use the subscripts Receiver and Budget to indicate whether a constraint is used to satisfy the physical requirements for converting an optical signal into an electronic signal at a receiver or is used to satisfy the budget requirements for remaining the signal in optical domain and continuing it in the next routing domain. 
a) Chromatic dispersion: $\mathrm{CD}$ is the dominant dispersion form in single-mode fibers (SMFs). It is caused because different frequency components of a pulse propagate through a fiber with different group velocities and arrive at the receiver at different times. The CD value is expressed as [39]

$$
\Delta t_{\text {output_CD }}=\Delta t_{\text {input_CD }}+\sum_{1 \leq k \leq M} D_{\mathrm{CD}}(k) \Delta \lambda L(k)
$$

where $M$ is the number of optical links along a local route, $k$ is the link index, $\Delta t_{\text {input_CD }}$ and $\Delta t_{\text {output_CD }}$ are the $\mathrm{CD}$ values of the entering and terminating optical signals on the route, respectively. $D_{\mathrm{CD}}(k)$ is the fiber CD parameter at the $k$ th optical link that has a length $L(k) . D_{\mathrm{CD}}(k)$ can be set to a wide range of values depending on what type of fiber and what kind of dispersion compensation technology are used. For fibers that use dispersion-shifted fiber (DSF), $D_{\mathrm{CD}}(k)$ is at most $3.3 \mathrm{ps} / \mathrm{nm} / \mathrm{km}$ in the $1.55 \mu \mathrm{m}$ wavelength range [40]. Note that many real-world network segments are still using standard SMF fibers and may not have any compensation, in which case $D_{\mathrm{CD}}(k)$ can be up to $20 \mathrm{ps} / \mathrm{nm} / \mathrm{km}$. In this paper we assume the former case. $\Delta \lambda$ is the optical signal spectral width in nanometers. We assume that $\Delta \lambda$ is equal to the modulation bandwidth (e.g., $2.5 \mathrm{GHz}$ for a $2.5 \mathrm{~Gb} / \mathrm{s}$ ON-OFF modulated signal, which is about $0.03 \mathrm{~nm}$ in the $1.55 \mu \mathrm{m}$ wavelength range) [40].

b) Polarization mode dispersion: PMD is caused by the time delay between two orthogonal polarizations of light traveling at different velocities through a fiber. The PMD value is expressed as [31]

$$
\Delta t_{\text {output_PMD }}=\sqrt{\Delta t_{\text {input_PMD }}^{2}+\sum_{1 \leq k \leq M} D_{\mathrm{PMD}}^{2}(k) L(k)}
$$

where $\Delta t_{\text {input_PMD }}$ and $\Delta t_{\text {output_PMD }}$ are the PMD values of the entering and terminating optical signals on the route. $D_{\mathrm{PMD}}(k)$ is the fiber PMD parameter at the $k$ th optical link that has a length $L(k)$. Typically, $D_{\mathrm{PMD}}(k)$ has a value ranging from $0.1 \mathrm{ps} / \sqrt{\mathrm{Km}}$ to $0.5 \mathrm{ps} / \sqrt{\mathrm{Km}}$ depending on the fiber technology used on the link [31].

c) Dispersion pool: In this paper, we place CD and PMD together into a dispersion pool so that they can share the same dispersion budget. By $\Delta t_{\text {input_dispersion }}$ and $\Delta t_{\text {output_dispersion, we denote the dispersions when }}$ the optical signal enters and leaves a routing domain. $\Delta t_{\text {input_dispersion }}=\Delta t_{\text {input_CD }}+\Delta t_{\text {input_PMD }}$ and $\Delta t_{\text {output_dispersion }}=\Delta t_{\text {output_CD }}+\Delta t_{\text {output_PMD }}$.

When an optical signal enters a local route through optical bypass, the value $\Delta t_{\text {input_dispersion }}>0$. If it enters the route through regeneration or electronic access, $\Delta t_{\text {input_dispersion }}=0$. If the signal undergoes regeneration at an interior node, $\Delta t_{\text {input_dispersion }}=0$ and only the optical links after the regeneration count.

When the signal terminates at the end of the local route, two different constraints can be imposed on the value of $\Delta t_{\text {output_dispersion. If the signal is dropped or regenerated at }}$ the access station of the local destination node $T$, we have the constraint

$$
\Delta t_{\text {output_dispersion }}<\frac{\alpha}{B}
$$

where $B$ is the digital bit rate of the signal and $\alpha$ is the maximum dispersion fraction in a bit interval that is acceptable for the receiver. $B$ can be $2.5,10$, or $40 \mathrm{Gbps}$ depending on the data rate. A typical value for $\alpha$ is 0.1 [31]. If the signal enters the route segment of a next domain through optical bypass, the following constraint will be imposed on this signal to guarantee that a predefined dispersion budget of the next domain is satisfied:

$$
\Delta t_{\text {output_dispersion }}<\Delta t_{\text {Budget_dispersion }}
$$

where $\Delta t_{\text {Budget_dispersion }}$ is the budget threshold, a constant value usually less than $\alpha / B$. In the latter case,

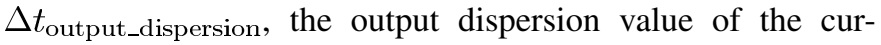
rent local route, will be equal to the input dispersion value ( $\left.\Delta t_{\text {input_dispersion }}\right)$ for the local route of the next domain.

d) Amplified spontaneous emission: The longer the path an optical signal is transported on, the more amplifiers (EDFAs) it needs to pass to compensate the power loss. The increased number of amplifiers makes the ASE noise a significant impairment factor to the signal quality. The ASE noise power on a local route can be expressed as [32]

$$
\begin{aligned}
& P_{\text {output_ASE }}=P_{1 \leq k \leq M} \sum_{j \in \operatorname{Link}(k)} n_{s p}(k, j)\left(G\left(\lambda_{k}, j\right)-1\right) h \nu_{k} B_{O} \\
& +\sum_{1 \leq \mathrm{SE}}
\end{aligned}
$$

where $P_{\text {input_ASE }}$ is the input ASE noise power when the signal enters the local route, $n_{s p}(k, j)$ and $G\left(\lambda_{k}, j\right)$ are the ASE factor and the saturated gain of the $j$ th amplifier on the $k$ th optical link along the route, respectively, $\lambda_{k}=c / \nu_{k}$ is the wavelength assigned to the $k$ th link along the route, $h$ is Planck's constant, $c$ is the velocity of light, and $B_{O}$ is the optical bandwidth.

At the local source node $S, P_{\text {input_ASE }}$ can be a value initialized either by a transmitter at the access station or by the signal entering from a previous route segment through optical bypass. Similar to dispersion, two different constraints can be imposed on $P_{\text {output_ASE }}$ at the terminating point. If the signal is dropped or regenerated at the local destination node $T$, $P_{\text {output_ASE }}$ should be below the detection threshold allowed by the receiver. Otherwise, if the signal enters the route segment of the next domain through optical bypass, a more stringent threshold should be used to satisfy the budget constraint on the ASE noise in the next domain.

e) Crosstalk: Crosstalk introduces nonlinear correlation between optical signals on different routes [33], which greatly increases the complexity of routing computation. In this paper, we only take into consideration the first-order crosstalks. Considering higher order crosstalk forms is complex. We can express the first-order crosstalk power on a local route with a linear equation

$$
\begin{array}{r}
P_{\text {output_Xtalk }}=P_{\text {input_XTALK }} \\
+\sum_{1 \leq k \leq M} \sum_{j \in \operatorname{Link}(k)} P_{\text {XTALK }}(k, j)
\end{array}
$$

where $P_{\text {input_XTALK }}$ is the initial crosstalk power introduced by the optical signal that enters the current local route (equal to zero if launched from a transmitter) and $\operatorname{PXtalk}(k, j)$ is 
the first-order crosstalk power introduced by the $j$ th component, e.g., an optical mux/demultiplexer [34] or a cross-connect [35], on the $k$ th optical link along the route. Note that the value of crosstalk power at different components is technology specific and/or vender specific. Even at the same component, the crosstalk may vary with traffic load. For example, at a cross-connect, when the load is heavy, there could be more first-order crosstalk sources interfering with each another than when the load is low. A solution for this variation is that only the worst case number of crosstalk sources is considered. This is reasonable because considering the worst case crosstalk at every component can avoid unexpected surge of total crosstalk power in the future. By summing up all the worst case crosstalks on each link, (6) is revised as

$$
P_{\text {output_XTALK }}=P_{\text {input_XTALK }}+\sum_{1 \leq k \leq M} P_{\text {XTALK }}(k)
$$

where $P_{\mathrm{XTALK}}(k)$ is the sum of the worst case crosstalk powers of all components on the k-th optical link. Again, the output crosstalk power $P_{\text {output_XTALK }}$ is subject to the constraint either on the detection threshold of the receiver if it is dropped to an access module or regenerated, or on the budget of the next domain if optical bypass is carried out.

f) Noise pool and Signal-to-Noise Ratio (SNR): The ASE noise power $P_{\text {output_ASE }}$ and the crosstalk power $P_{\text {output_Xtalk }}$ can share the same noise pool, which is determined by the output signal power and the minimum SNR allowed at the local destination node $T$. We have assumed that the signal power is constant. Therefore, the constraints on ASE noise power and crosstalk power can be integrated into the constraints on SNR. By $P_{\text {Signal }}$ we denote the signal power. By SNR $_{\text {Receiver_min }}$, we denote the minimum SNR allowed by the receiver when regeneration or access is carried out. By $\mathrm{SNR}_{\text {Budget_min }}$, we denote the minimum SNR allowed by the budget of the next domain when optical bypass is carried out. The SNR constraints on the detection threshold of the receiver and on the budget of the next domain are expressed, respectively, as follows:

$$
\begin{gathered}
\frac{P_{\text {Signal }}}{\left(P_{\text {output_ASE }}+P_{\text {output_XTALK }}\right)}>\text { SNR }_{\text {Receiver_min }} \\
\frac{P_{\text {Signal }}}{\left(P_{\text {output_ASE }}+P_{\text {output_XTALK }}\right)}>\text { SNR }_{\text {Budget_min }}
\end{gathered}
$$

2) Constraints on Wavelength Continuity: Wavelength continuity is the constraint that restricts an optical signal to be transported using the same wavelength in optical domain. We assume that no optical wavelength converter is available in translucent networks and wavelength conversion must be carried out through $\mathrm{O} / \mathrm{E} / \mathrm{O}$ regeneration.

A single wavelength should be assigned to the same optical signal on two consecutive optical links. This is expressed as a chain of constraints

$$
w(k)=w(k-1), \quad 1<k \leq M
$$

where $w(k)$ is the wavelength assigned on the $k$ th optical link and $M$ is the total number of optical links on the local route.

When regeneration is carried out at the $r$ th intermediate node of the local route, the constraint chain is broken into two constraint chains, which are expressed as

$$
w(k)=w(k-1), 1<k \leq r \quad \text { and } \quad w(l)=w(l-1), r<l \leq M .
$$

\section{Formulation}

We formulate the problem of intradomain dynamic routing in a translucent network as follows.

\section{1) Notation:}

$G=(V, E)$

\section{$S, T$}

W

$\operatorname{Set}_{W}$

$w_{S}, w_{T}$

$x$

$\operatorname{Set}_{R}$

$\Omega_{\mathrm{TR}}(i)$

$\operatorname{Set}_{\mathrm{EI}}(i)$

Role

$\operatorname{IPT}(i)$

2) Inputs:

- A network topology $G=(V, E)$ is given with predefined boundary nodes and interior nodes. We refer to each fiber as a fiber link. Two fiber links on the same link are treated separately. Each link has a pair of fibers in opposite directions. Each fiber link has $W$ wavelengths in Set $_{W}$. At each regeneration capable node $i$, a predefined number of regeneration resources (represented by $\Omega_{\mathrm{TR}}(i)$ and $\left.\operatorname{Set}_{\mathrm{EI}}(i)\right)$ are assigned.

- A local route from $S$ to $T$ is requested. The optical signal starting at $S$ uses the wavelength $w_{S}$ while the optical signal terminating at $T$ uses the wavelength $w_{T}$. The optical signals carry a digital signal of format type $x$. Note that instead of starting and terminating with arbitrary wavelengths and arbitrary types of digital format, a local route in our formulation starts and terminates with specified wavelengths and a specified type of digital 
format. This is particularly useful for interdomain routing to request the intradomain routing algorithm to provide local route segments with desired wavelengths and types of digital format when setting up a lightpath.

- A Role is requested for the local route to serve in the lightpath (see Section IV-A).

- The initial impairment parameter triplet $\operatorname{IPT}(S)$ is set to be $\langle 0,0,0\rangle$, if electronic access or regeneration is carried out at $S$. If the signal enters $S$ through optical bypass, $\operatorname{IPT}(S)$ is set to be $\left\langle\Delta t_{\text {input_dispersion }}, P_{\text {input_ASE }}, P_{\text {input_XTALK }}\right\rangle$, the actual impairment values of the entering signal from the previous domain. IPT $(i)$ is computed for each intermediate node $i$ along the local route.

$\operatorname{IPT}(T)=$

$$
\left\langle\Delta t_{\text {output_dispersion }}, P_{\text {output_ASE }}, P_{\text {output_XTALK }}\right\rangle
$$

representing the output IPT at $T$. IPT $(i)$ and $\operatorname{IPT}(T)$ can be computed using (1), (2), (5), and (7).

3) Constraints:

- At the local destination node $T$, if the signal is dropped or regenerated, the output IPT values $\Delta t_{\text {output_dispersion, }}$ $P_{\text {output_ASE }}$, and $P_{\text {output_XTALK }}$ should satisfy the constraint Inequalities (3) and (8), i.e. the constraints on the detection threshold of the receiver. Otherwise, if the signal is transported to a next route segment through optical bypass, the output IPT values should satisfy the constraint Inequalities (4) and (9), i.e., the constraints on the impairment budget of the next domain.

- The optical signal on the local route must satisfy (10), i.e., the wavelength continuity constraint chain, unless the wavelength is converted at some intermediate node through regeneration, which implies the alternative constraint (11).

- At most one regeneration, i.e., regeneration at an interior regeneration capable node $R$ other than $S$ and $T$, is allowed. This regeneration at the node $R$ will reset the values of $\operatorname{IPT}(R)$ to zeros and break the wavelength continuity constraint chain into two constraint chains (11). Based the experimental results in our previous work [5], we assume that one regeneration at an interior node plus regeneration at boundary nodes is sufficient for routing any signal within a single domain.

- For each regeneration at $S, T$ and an intermediate interior regeneration capable node, a pair of electronic interfaces that support the digital format type $x$ must be allocated.

4) Objectives: Find a local route for the optical signal from the node $S$ to $T$ using all the inputs in Section IV-C2 and satisfying all the constraints in Section IV-C3. The following objectives are desired in the descending order of priority:

- minimizing the number of regenerations, i.e., the regenerations at $S, T$ and an intermediate node;

- minimizing the number of optical links on the route;

- minimizing the IPT values at $T$.

Minimizing the number of regenerations takes the highest priority because the transmitters, receivers, and electronic interfaces needed for regeneration are expensive resources. Objective 2 minimizes the use of optical layer resources. Objective 3 minimizes the physical impairments. For objective 3, the comparison of two IPTs, say,

$$
\mathrm{IPT}^{\prime}(T)=\left\langle\Delta t_{\text {output_dispersion }}^{\prime}, P_{\text {output_ASE }}^{\prime}, P_{\text {output_XTALK }}^{\prime}\right\rangle
$$

and

$\mathrm{IPT}^{\prime \prime}(T)=\left\langle\Delta t_{\text {output_dispersion }}^{\prime \prime}, P_{\text {output_ASE }}^{\prime \prime}, P_{\text {output_XTALK }}^{\prime \prime}\right\rangle$

follows two rules: if $P_{\text {Signal }} /\left(P_{\text {output_ASE }}^{\prime}+P_{\text {output_ASE }}^{\prime}\right)$ $\left\langle P_{\text {Signal }} /\left(P_{\text {output_ASE }}^{\prime \prime}+P_{\text {output_ASE }}^{\prime \prime}, \mathrm{IPT}^{\prime}(T)\right\rangle \mathrm{IPT}^{\prime \prime}(T)\right.$ (compare their SNR values); if the SNR values are equal and $\Delta t_{\text {output_dispersion }}^{\prime}>\Delta t_{\text {output_dispersion }}^{\prime \prime} \operatorname{IPT}^{\prime}(T)>\operatorname{IPT}^{\prime \prime}(T)$ (compare their dispersion values). Comparing SNR takes priority over comparing dispersion because dispersion is usually easier to handle than noise.

\section{InTRADOMAIn Dynamic Routing ALgORITHM}

As formulated, some optical-layer constraints introduce nonlinear relations between different routes and even between links along the same route so that a constrained shortest path first (CSPF) routing algorithm, which requires linear constraints, cannot be applied. Due to the same reason, the often-used linear programming (LP) approach is also not applicable. We resort to a breadth-first search (BFS) based heuristic routing algorithm, called intradomain dynamic balancing routing (IDDBR) algorithm, to provide a practical solution.

\section{A. OLC-BFS Procedure}

We introduce a procedure called optical-layer constrained breadth-first search (OLC-BFS) to facilitate the IDDBR algorithm. Subject to the optical-layer constraints described in Section IV-B, the OLC-BFS procedure searches at the optical layer (i.e., without regeneration) for a shortest routing path using the wavelength $w$ and the digital format type $x$. The basic idea is to use BFS to explore alternate routes from the source $S$ to the destination $T$. When OLC-BFS proceeds, the wavelength continuity constraint (10) must be satisfied and the IPT values $\Delta t_{\text {output_dispersion, }}, P_{\text {output_ASE }}$, and $P_{\text {output_XTALK }}$ at the end of each route are computed online using (1), (2), (5), and (7). When two or more explored routes meet at the same intermediate node, the routes that have traversed more optical links are discarded. If the numbers of traversed optical links are equal, those with higher IPT values are discarded (see Section IV-C4 for information on how to compare two IPTs). When the destination $T$ is reached by an explored route, the IPT values are tested using the constraint inequalities (3) and (8) or (4) and (9), depending on whether access/regeneration or optical bypass is carried out. Finally, the route with the least number of optical links (or lowest IPT values if the numbers of optical links are equal) is selected among all the routes that have passed the test. If no route is selected, the OLC-BFS procedure fails. The flowchart of the OLC-BFS procedure is shown in Fig. 3.

\section{B. IDDBR Algorithm}

We design the IDDBR algorithm based on the formulation described in Section IV. It is divided into five steps. In the first 


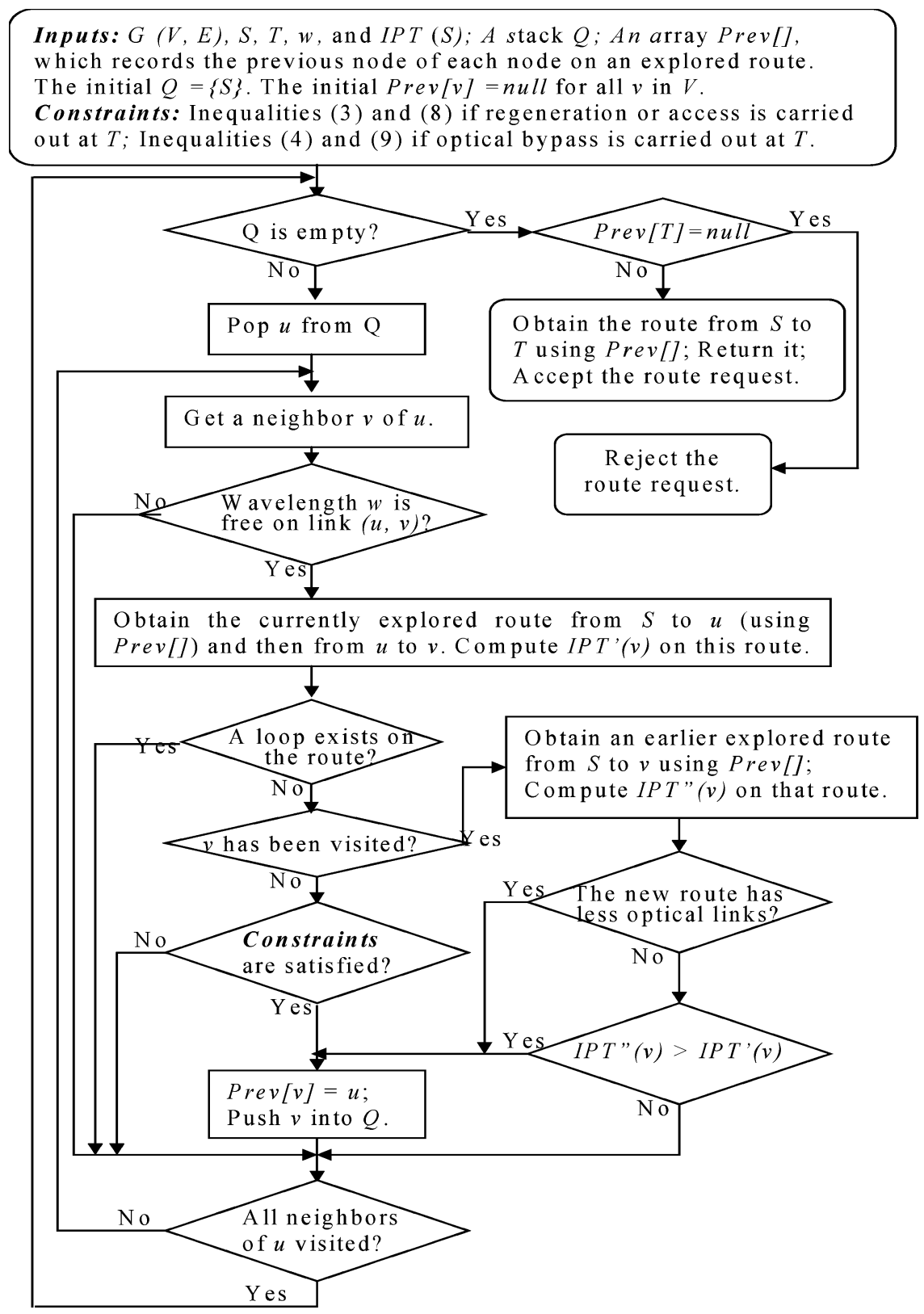

Fig. 3. Flowchart of the OLC-BFS procedure.

step, we try to find an all-optical route, along which optical bypass is carried out at every node. In each of the following three steps, more regenerations, at the node $S$, an intermediate interior regeneration capable node, and/or the node $T$, are used to facilitate candidate routes that satisfy all the constraints formulated in Section IV-C3. The OLC-BFS procedure is iteratively invoked by these steps to search for the route segments along each candidate route. In the last step, a route among all those candidate routes is selected. This algorithm is called dynamic balancing because, in addition to the three objectives formulated in Section IV-C4, it tries to balance the allocation of regeneration resources between boundary nodes and interior regeneration capable nodes. Details of the IDDBR algorithm are described below.

Step 1) Trying optical bypass at all nodes: If the wavelength $w_{S} \neq w_{T}$, go to Step 2). If $w_{S}=w_{T}$, the algorithm tries to find a transparent route optically bypassing $S, T$, and all intermediate nodes. The IPT at node $S$ is initialized as

$\left\langle\Delta t_{\text {input_dispersion }}, P_{\text {input_ASE }}, P_{\text {input_XTALK }}\right\rangle$.

Under the constraint inequalities (4) and (9), the OLC-BFS procedure is invoked to search for a route from $S$ to $T$ using wavelength $w_{S}$.

Step 2) Trying regeneration either at $S$ or at an intermediate interior node $R$ : If Step 1) fails, IDDBR resorts to regeneration. Based on the dynamically disseminated routing information, the algorithm computes the average number of $\mathrm{T}-\mathrm{R}$ pairs and the average number of electronic interfaces of digital format type $x$ that are dynamically allocated as regeneration resources at all interior regeneration capable nodes in $\operatorname{Set}_{R}$ 
(other than $S$ if applicable). If on average there are more regeneration resources (relative to their total numbers) available at an intermediate interior regeneration capable node in $\operatorname{Set}_{R}$ than at $S$, regeneration is carried out at one of those nodes and the signal optically bypasses $S$. This condition is expressed by the following two inequalities:

$$
\begin{aligned}
& \frac{\eta}{\left|\operatorname{Set}_{R}-\{S\}\right|} \sum_{i \in \operatorname{Set}_{R}, i \neq S} \Omega(i)>\Omega(S) \\
& \frac{\rho}{\left|\operatorname{Set}_{R}-\{S\}\right|} \sum_{i \in \operatorname{Set}_{R}, i \neq S}\left|\left\{x \in \operatorname{Set}_{E I}(i)\right\}\right| \\
& \quad>\left|\left\{x \in \operatorname{Set}_{E I}(S)\right\}\right|
\end{aligned}
$$

where $\Omega(i)$ has been defined as the number of spare T-R pairs at the node $i,\left|\left\{x \in \operatorname{Set}_{E I}(i)\right\}\right|$ represents the number of spare electronic interfaces supporting the digital format type $x$ at the node $i, \eta$ is the ratio of the total number of T-R pairs at $S$ to the total number of T-R pairs at an interior regeneration capable node, and $\rho$ is the ratio of the total number of electronic interfaces at $S$ to the total number of electronic interfaces at an interior regeneration capable node. When $S$ is an interior regeneration capable node, both $\eta$ and $\rho$ are equal to one. When $S$ is a boundary node, $\eta$ and/or $\rho$ may not be equal to one, because the total number of regeneration resources initially assigned to a boundary node may be different from that assigned to an interior regeneration capable node.

When both (12) and (13) are satisfied, an interior regeneration capable node $R$ is tried as an intermediate regeneration node for this route. Multiple iterations of the OLC-BFS procedure, each trying a different $R$ in Set ${ }_{R}$, are invoked to search for two route segments from $S$ to $R$ and from $R$ and $T$, respectively. For the segment from $S$ to $R$, the wavelength $w_{S}$ is used. For the segment from $R$ to $T$, the wavelength $w_{T}$ is used. If one of the two inequalities (12) or (13) is false, the regeneration is carried out at the node $S$, while the signal is transparently transported from $S$ to $T$ and optically bypasses $T$. In this case, the wavelength $w_{T}$ from $S$ to $T$ is used. Regeneration at $S$ needs at least a T-R pair and an electronic interface of digital format type $x$, i.e.,

$$
\begin{aligned}
\Omega(S) & >0 \\
\left|\left\{x \in \operatorname{Set}_{E I}(S)\right\}\right| & >0 .
\end{aligned}
$$

Step 3) Trying regeneration both at $S$ and at an intermediate interior node $R$ : If Step 2) also fails, IDDBR allows regeneration both at $S$ and at an intermediate interior regeneration capable node. Every interior regeneration capable node $R$ in $\operatorname{Set}_{R}$ (other than $S$ and $T$ if applicable) is examined as a candidate regeneration node. Each wavelength in $\operatorname{Set}_{W}$ is used by OLC-BFS to search for the route segment from $S$ to $R$, because regeneration allows for wavelength con- version at both $S$ and $R$. For the segment from $R$ to $T$, only wavelength $w_{T}$ is used.

Step 4) Trying regeneration at $T$ : If Step 3) fails, no route can be found to allow for optical bypass at the node $T$. The algorithm checks if regeneration resources for this signal are available at the node $T$ using the following two inequalities:

$$
\begin{aligned}
\Omega(T) & >0 \\
\left|\left\{x \in \operatorname{Set}_{E I}(T)\right\}\right| & >0 .
\end{aligned}
$$

If (15) and (16) are true, regeneration instead of optical bypass is carried out at the node $T$. The constraints on the IPT values are relaxed to the constraint inequalities (3) and (8), which are required by the detection threshold of the receiver. Then, Steps 1)-3) are repeated under these new constraints. There is another minor difference in the new round of execution of Steps 2) and 3). When searching for the route segment from $S$ to $T$ and the route segment from $R$ to $T$, each wavelength in $\operatorname{Set}_{W}$ should be tried by the OLC-BFS procedure because regeneration allows for wavelength conversion at both $S$ and $T$ and at both $R$ and $T$.

Step 5) Choice among candidate routes: If Step 4) fails, the algorithm returns and rejects the local route request from $S$ to $T$. Success in any of the Steps 1-4) results in one or several routes. If the route is requested to serve the role $A$ (see Section IV-A) in a lightpath, all these routes are candidate routes. If the route serves the role $B$, only the routes with regeneration at $S$ are candidate routes. If the route serves the role $C$, only the routes with regeneration at $T$ are candidate routes. If the route serves the role $D$, only the routes with regeneration at both $S$ and $T$ are candidate routes. For any of the four roles, if more than one candidate route is obtained, the preference of routes follows the step order 1) $>2$ ) $>3$ ) $>4$ ). If the preferred step still results in more than one candidate route, the route with the lowest IPT is chosen.

In Step 2), a balancing resource allocation procedure is used to decide whether to carry out regeneration at the local source node $S$ or at an intermediate interior regeneration capable node $R$ based on their regeneration resource availability status. The local source node $S$ is usually a boundary node, which performs regeneration only if it has more than the average numbers of regeneration resources at those interior regeneration capable nodes. In this regard, the IDDBR algorithm can not only dynamically establish local routes with appropriate regeneration under optical-layer constraints but also guarantee balanced allocation of regeneration resources between the boundary nodes and the interior regeneration capable nodes. Step 5) ensures that the selected local route serves the desired role in a lightpath and the optimization objectives are satisfied ac- 


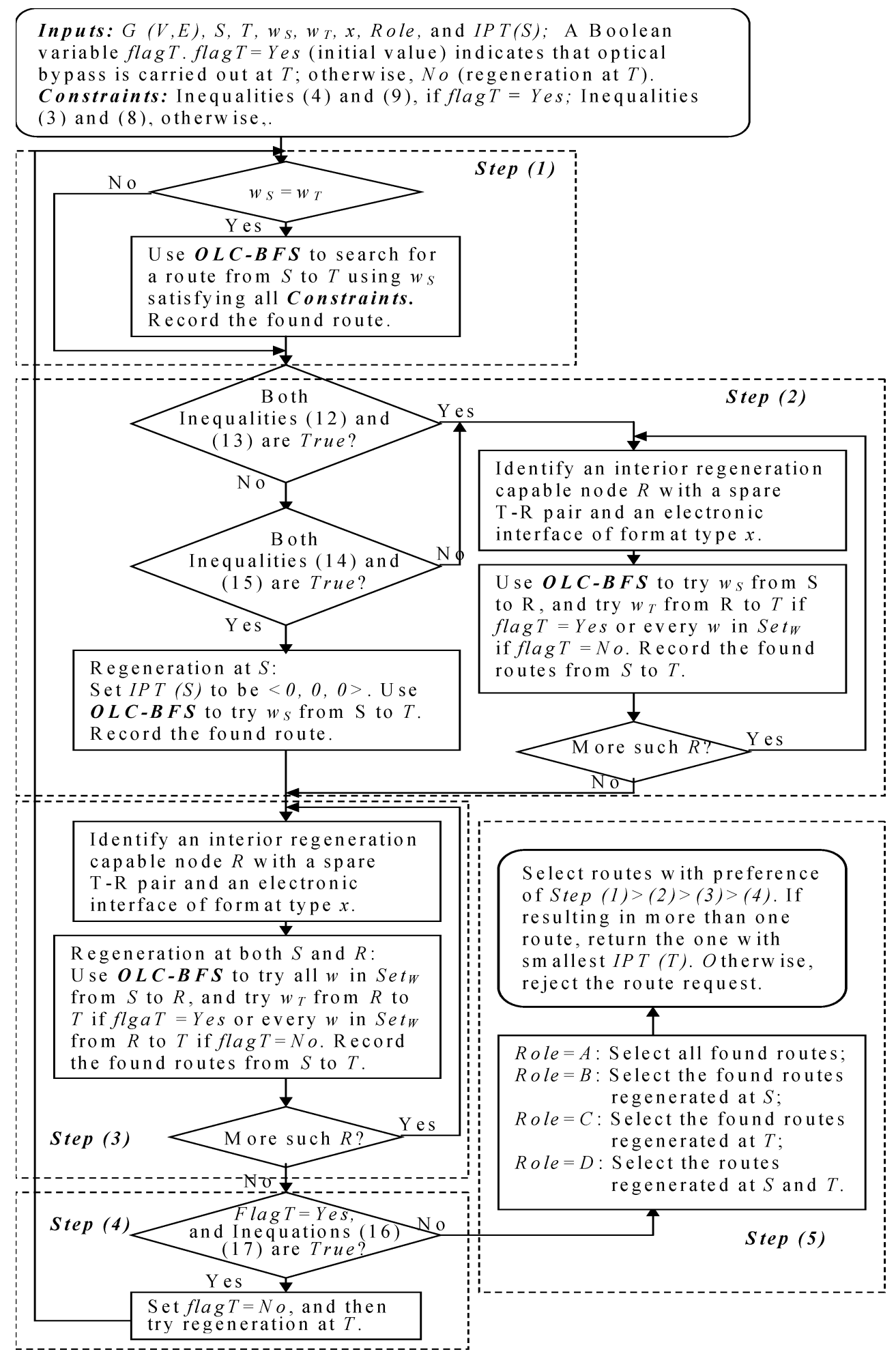

Fig. 4. Flowchart of the IDDBR algorithm.

cording to their order of priority. The flowchart of the IDDBR algorithm is shown in Fig. 4.

\section{Time Complexity}

Similar to a regular BFS algorithm, OLC-BFS has a time complexity $\mathrm{O}(|E|+|V|)[36]$, because BFS only searches at the optical layer using one wavelength and one type of digital format, where $|E|$ is the number of optical links and $|V|$ is the number of nodes in the domain. IDDBR has a varying time complexity depending on the alternate routes it tries. In the worst case, every interior regeneration capable node $R$ in $\operatorname{Set}_{R}$ (other than $S$ and $T$ if applicable) is tried as an intermediate regeneration node and at least two nodes out of $S, T$, and $R$ are selected as regeneration nodes, so that all wavelengths 


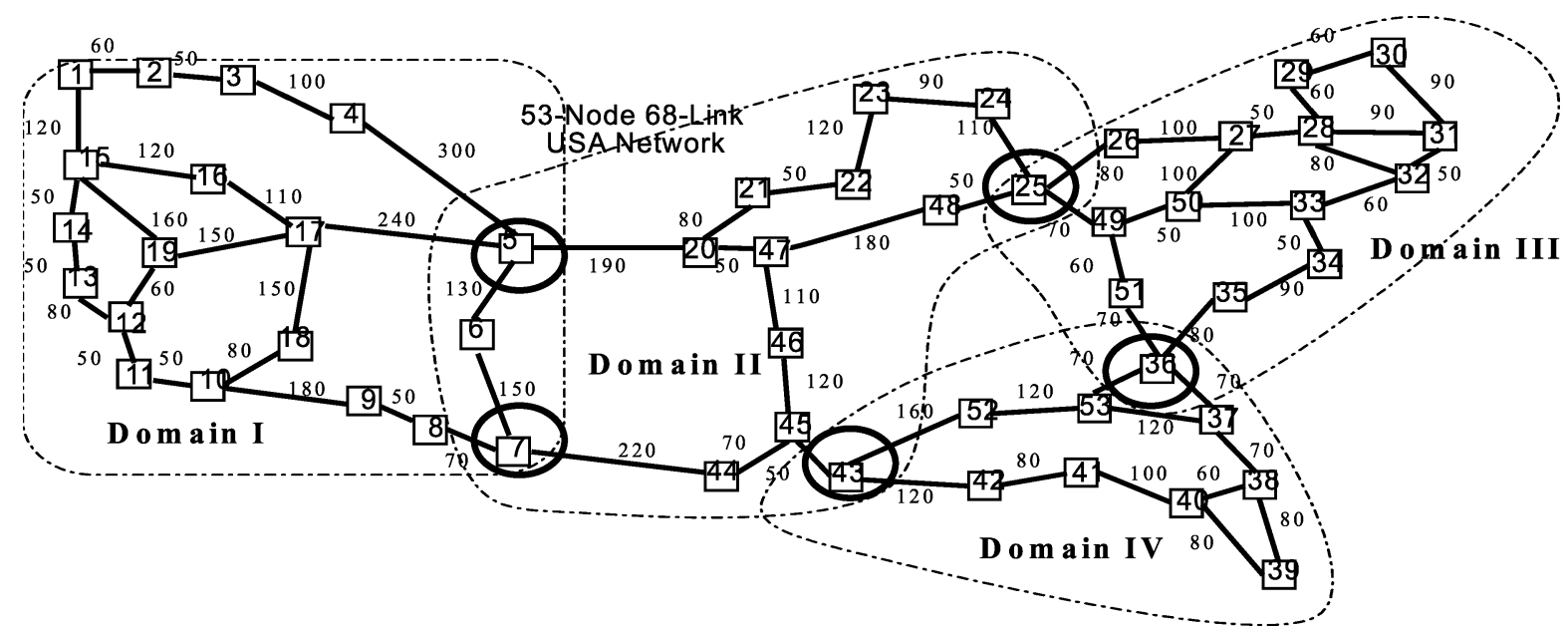

Fig. 5. Topology of the 53-node 68-link network, which is partitioned into four domains: I, II, III, and IV. The circled nodes are boundary nodes. All other odd numbered nodes are interior regeneration capable nodes and all other even numbered nodes are interior transparent nodes. A link length is labeled on each link.

TABLE I

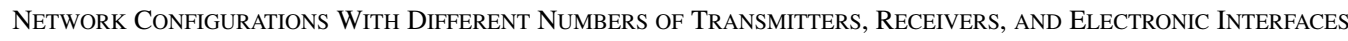

\begin{tabular}{l|c|c|c|c|c|c}
\hline \multirow{2}{*}{$\begin{array}{c}\text { Category of } \\
\text { Resources and } \\
\text { Nodes }\end{array}$} & $\begin{array}{c}\text { Number of } \\
\text { T-R pairs at } \\
\text { each interior } \\
\text { regeneration } \\
\text { capable node }\end{array}$ & $\begin{array}{c}\text { Number of } \\
\text { T-R pairs at } \\
\text { each boundary } \\
\text { node }\end{array}$ & $\begin{array}{c}\text { Number of electronic } \\
\text { interfaces at each } \\
\text { interior regeneration } \\
\text { capable node }\end{array}$ & $\begin{array}{c}\text { Number of electronic } \\
\text { interfaces at each } \\
\text { boundary node }\end{array}$ \\
\cline { 4 - 8 } & & 4 & 4 & 4 & 4 & 4 \\
\hline \hline Configuration-1 & 4 & 8 & 8 & 8 & 8 & 8 \\
\hline Configuration-2 & 8 & 8 & 4 & 4 & 8 & 8 \\
\hline Configuration-3 & 4 & 16 & 8 & 8 & 16 & 16 \\
\hline Configuration-4 & 8 & & & & & \\
\hline
\end{tabular}

in Set $_{W}$ are tried by using OLC-BFS between these two regeneration nodes. Therefore, the worst case time complexity is $\mathrm{O}\left((|E|+|V|) \cdot W \cdot\left|\operatorname{Set}_{R}\right|\right)$, where $W=\left|\operatorname{Set}_{W}\right|$ is the number of wavelengths supported on each fiber link and $\left|\operatorname{Set}_{R}\right|$ is the number of interior regeneration capable nodes in the domain. If $|V| \leq E \mid$ and $\left|\operatorname{Set}_{R}\right| \leq|V|$, the time complexity is equal to $\mathrm{O}(|E| \cdot W \cdot|V|)$.

\section{EXPERIMENTAL RESULTS}

\section{A. Experiment Design}

In this section, we conduct simulation experiments on a largescale translucent network to measure the network performance under different resource allocation and routing schemes. The topology of the network, as shown in Fig. 5, is formed by selecting 53 representative nodes and 68 corresponding bidirectional links from the Global Crossing network topology [37] that was being operated in the real world. Each node in the network contains a $64 \times 64$ OXCs and an access station. Each link supports 16 wavelengths in each direction. The length of a link ranges from 50 to $300 \mathrm{~km}$ depending on its location in the topology. We assume that EDFAs are placed every $50 \mathrm{~km}$ along a fiber link. The five circled nodes in the topology are boundary nodes, which partition the network into four domains: I, II, III, and IV. Each remainder node is an interior node in one of the domains. Each odd numbered interior node is a regeneration capable node, while each even numbered interior node is a transparent node. Our experiments are conducted under four different kinds of network configurations, each assigning different numbers of transmitters, receivers, and electronic interfaces to boundary nodes and interior regeneration capable nodes. These configurations are described in Table I. Each interior transparent node has four transmitters, four receivers, and four GigE and four STM-16 electronic interfaces, which are only used to perform the access function.

The intradomain traffic of each domain is derived from the traffic across the entire network. We generate uniformly distributed traffic between every pair of nodes in the network. The traffic is converted into wavelength-level end-to-end lightpath requests. Such requests arrive following a Poisson distribution with exponential holding times. By changing the ratio of holding time to interarrival time, the total amount of traffic is increased from one erlang, defined as one wavelength per lightpath per hour, to a value high enough to cause severe blocking in the network. Two types of digital format, GigE (1 Gbps) and STM-16 (2.5 Gbps), are carried on these lightpaths in a half-to-half proportion randomly. A lightpath is divided into one or more route segments with each assigned to a corresponding domain. The domains that the lightpath traverses are determined by using an unconstrained SPF routing algorithm. According to its position in the lightpath, a route segment is assigned one of the four roles as described at Section IV-A. For each of those route segments, the wavelengths $w_{S}$ and $w_{T}$ at the local source $S$ and destination $T$ are assigned at random from $\operatorname{Set}_{W}$. We do not need to specify which wavelengths should be used when starting and terminating a specific route segment, because we are not concerned with interdomain routing in this paper. Each derived route segment introduces into its corresponding domain a local 
TABLE II

SYSTEM PARAMETERS AND THEIR VALUES USED IN THE EXPERIMENTS

\begin{tabular}{|c|c|c|c|}
\hline Parameter & Value & Parameter & Value \\
\hline Data rate $(B)$ & 1or $2.5 \mathrm{Gbps}$ & Wavelength spacing & $100 \mathrm{GHz}$ \\
\hline Wavelength $\left(\lambda_{k}\right)$ range & $1530-1565 \mathrm{~nm}$ & Fiber attenuation factor & $.2 \mathrm{~dB} / \mathrm{km}$ \\
\hline Bit error rate (BER) threshold & $10^{-12}$ & Fiber CD coefficient $\left(\mathrm{D}_{\mathrm{CD}}(k)\right.$ for any $\left.k\right)$ & $\mathrm{ps} / \mathrm{nm} / \mathrm{km}$ \\
\hline \multirow{2}{*}{$\begin{array}{l}\text { Fiber PMD parameter }\left(D_{P M D}(k) \text { for }\right. \\
\text { any } k)\end{array}$} & \multirow{2}{*}{0.2} & Output signal power EDFA $\left(P_{\text {Signal }}\right)$ & $V(0 \mathrm{dBm})$ \\
\hline & & Optical bandwidth $\left(B_{O}\right)$ & $50 \mathrm{GHz}$ \\
\hline ASE factor $\left(n_{s p}(k)\right.$ for any $\left.k\right)$ & 1.5 & EDFA saturated gain $\left(G\left(\lambda_{k}\right)\right.$ for any $\left.\lambda_{k}\right)$ & $21 \mathrm{~dB}$ \\
\hline Mux/Demultiplex crosstalk ratio & $-25 \mathrm{~dB}$ & First-order switch crosstalk ratio & $-25 \mathrm{~dB}$ \\
\hline $\begin{array}{l}\text { Maximum number of mux/demultiplex } \\
\text { crosstalk sources per link }\end{array}$ & $\mathrm{x}$ & $\begin{array}{l}\text { Maximum number of first-order switch } \\
\text { crosstalk sources per node }\end{array}$ & 3 \\
\hline $\begin{array}{l}\text { Maximum dispersion at a receiver } \\
\text { for access or regeneration }(\alpha / B) \\
\end{array}$ & 100 or $25 \mathrm{ps}$ & $\begin{array}{l}\text { Maximum dispersion for optical signals entering } \\
\text { a next domain through optical bypass }(\alpha / 2 B)^{\$}\end{array}$ & $\begin{array}{l}50 \text { or } \\
12 \text { ps } \\
\end{array}$ \\
\hline $\begin{array}{l}\text { Minimum SNR at a receiver for access } \\
\text { or regeneration }\left(S N R_{\text {Receiver } \min }\right) \\
\end{array}$ & $20 \mathrm{~dB}$ & $\begin{array}{l}\text { Minimum SNR budget for optical bypass } \\
\left(\text { SNR } R_{\text {Budget min })}\right.\end{array}$ & $25 \mathrm{~dB}$ \\
\hline
\end{tabular}

$\left({ }^{\$}\right.$ We set the budget threshold $\Delta t_{\text {Budget_dispersion }}$ to be as a half of the value $\alpha / B$ (see Inequalities (3) and (4)))

route request that consists of the local source and destination node identifiers $(S$ and $T$ ), the type of digital format $(x)$, the wavelengths at the local source and destination $\left(w_{S}\right.$ and $\left.w_{T}\right)$, and the role $($ Role $)$ in a lightpath.

The system parameters used in the experiments are shown in Table II. We assume uniform system parameter values for all links, nodes and components. We also assume uniform $\mathrm{SNR}_{\text {Receiver_min }}$ and $\mathrm{SNR}_{\text {Budget_min }}$ values for the signals using different types of digital format. Note that the uniformity assumption is only to simplify the experiment setting while our algorithms can handle nonuniform parameters.

The simulation experiments are conducted using a proven tool: the simulator for optical networks (SIMON) [38]. SIMON has embedded physical models for a variety of components and supports online computation of dispersion, ASE noise power, and crosstalk power. Details about those models and computation methods can be found in [33]. In this paper, SIMON is enhanced to support dynamic sharing of transmitters, receivers, and electronic interfaces between access and regeneration functions. It is also enhanced to support the IDDBR algorithm. The enhanced SIMON takes IPT parameters such as dispersions and noises to compute the bit error rate (BER) value for each connection request. A connection request is blocked if the BER value goes above the BER threshold or if wavelength continuity constraint cannot be satisfied. In each round of experiment, we use the method described above to generate 100000 wavelength-level end-to-end lightpaths, derive corresponding local route requests, and assign the local route requests to the corresponding domains. We use three kinds of resource allocation and routing schemes below to handle these derived local route requests in every domain, collect statistics, compare results, and present the analysis.

1) Static resource allocation and static routing (SASR) scheme: In this scheme, a fixed fraction of transmitters, receivers, and electronic interfaces are statically allocated at each regeneration capable node to perform the regeneration function. The remainder transmitters, receivers, and electronic interfaces are used to perform the access function. The fraction value is set to $1 / 4$ and $1 / 2$, corresponding to the schemes SASR-1/4 and SASR-1/2, respectively. The unconstrained SPF routing and first-fit wavelength assignment algorithm is used. When an SPF route cannot satisfy the constraints on physical impairments and wavelength continuity, regeneration is tried at some intermediate nodes using the fragmentation method described in [5], which simply carries out regeneration at the first intermediate node where signal quality degrades below a threshold or where wavelength contention occurs.

2) Dynamic resource allocation and static routing (DASR) scheme: In DASR, transmitters, receivers, and electronic interfaces are allowed to be dynamically shared between the access and regeneration functions. The same static routing, wavelength assignment, and regeneration methods as in SASR are used.

3) Dynamic resource allocation and dynamic routing (DADR-IDDBR) scheme: DADR is the scheme we proposed in this paper. DADR uses the IDDBR algorithm in each routing domain to search for appropriate routes under optical-layer constraints and allocate corresponding regeneration resources dynamically.

\section{B. Blocking Probability}

Fig. 6 shows the blocking probability of intradomain routing under four different kinds of network configurations. The two SASR schemes result in higher blocking probability than other schemes under all configurations. Under heavy traffic loads, the blocking probability of SASR reaches 0.5 , a higher value than most of those obtained by previous studies that used similar static schemes. This increase in blocking probability can be explained by the physical impairment constraints we introduced into the routing problem. Stricter physical impairment constraints inevitably cause greater blockings. We can also observe that SASR-1/2 outperforms SASR-1/4 because the former allocates more resources to perform the regeneration function.

The DADR-IDDBR scheme yields the best performance under all configurations as well as under both light and heavy traffic loads. It is particularly remarkable that even under heavy traffic loads, DADR-IDDBR still provides a stable and acceptable performance. This is due to its sophisticated search procedure under optical-layer constraints and its capability of balancing regeneration resource allocation. 


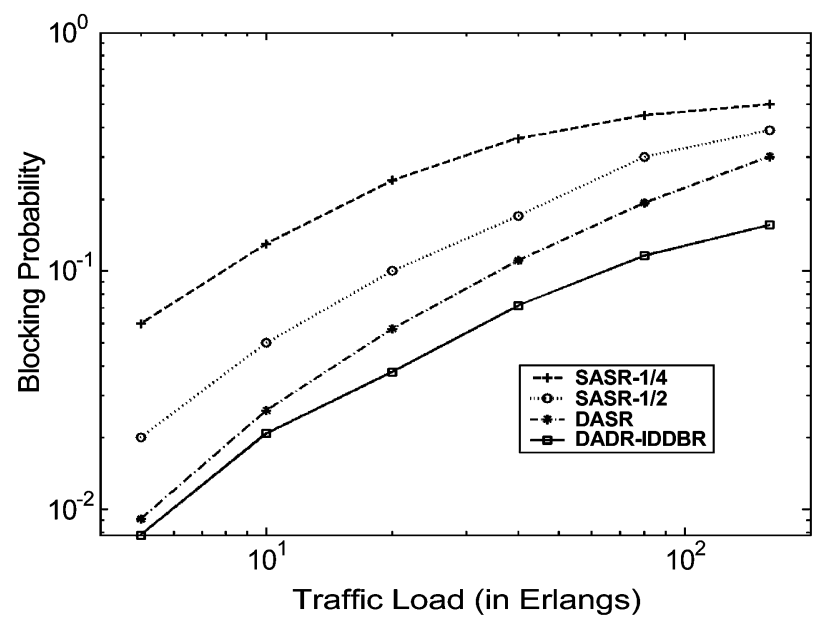

(a)

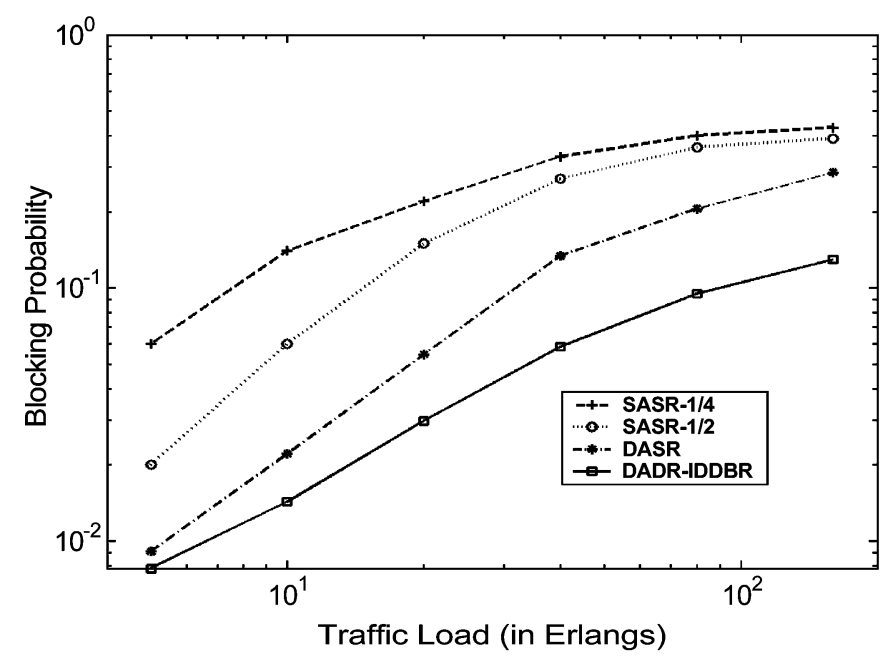

(c)

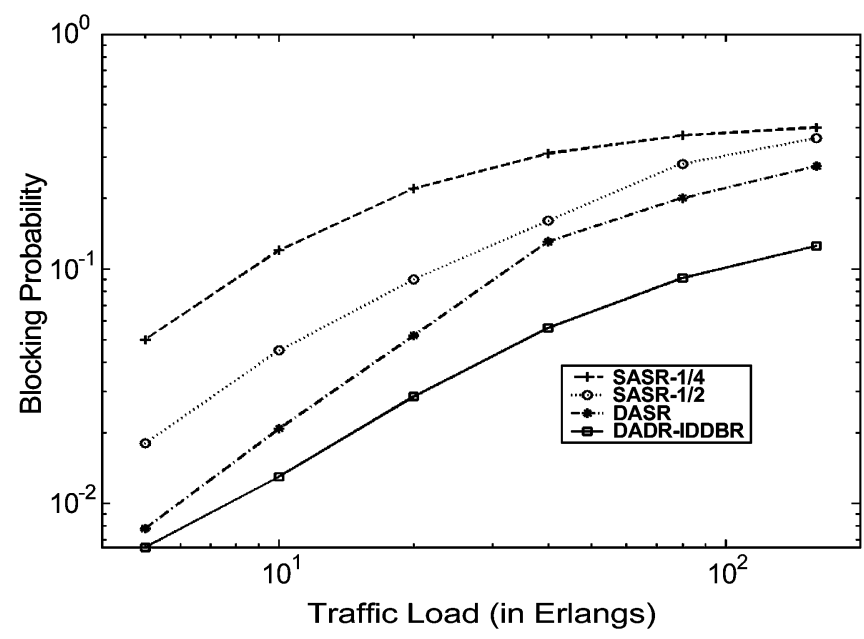

(b)

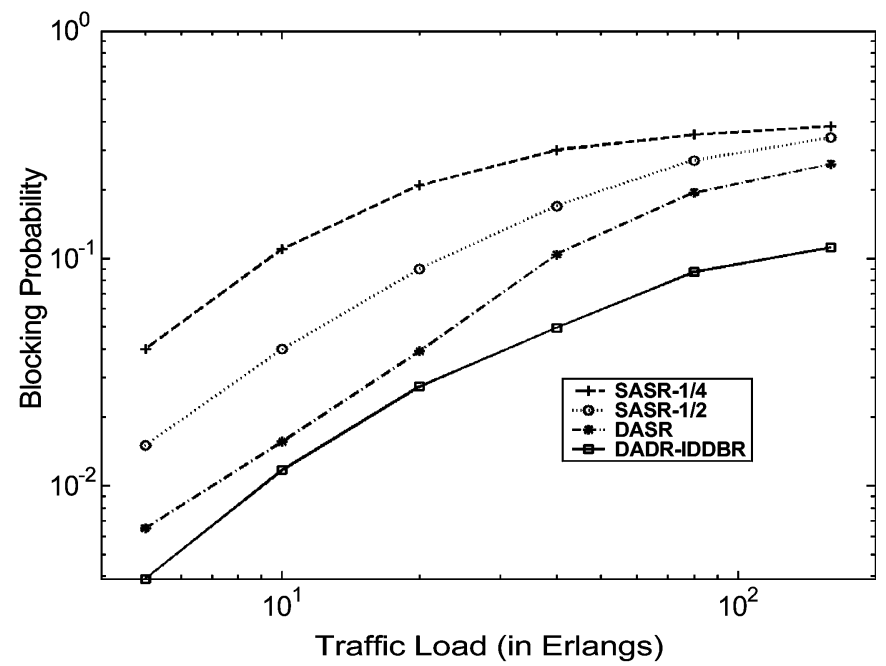

(d)

Fig. 6. Blocking probability versus traffic load under different network configurations. (a) Configuration-1; (b) Configuration-2; (c) Configuration-3; and (d) Configuration-4.

DASR also shows a good performance, especially when the traffic load is light. This suggests that sharing transmitters, receivers, and electronic interfaces between the access and regeneration functions has a significant benefit. However, when the traffic load becomes heavy, DASR causes a high blocking probability only slightly lower than the SASR schemes. This suggests that static routing and wavelength assignment algorithms cannot meet the performance requirement for operation of a translucent network. Dynamic routing algorithms aware of regeneration resources, such as the IDDBR algorithm, are very desirable.

\section{Resource Utilization}

Table III shows the resource utilization in percentage for the entire network under Configuration-2, which allocates the same numbers of regeneration resources to each interior regeneration capable node as to each boundary node. The statistics are collected by considering the utilization of different categories of network resources when a predefined total number of local route requests are admitted in all domains. The average (AVG) link utilization is defined as $(1 /|E|) \sum_{l}\left(u_{l} / W\right), l \in E$, where $u_{l}$ is the number of used wavelengths on link $l$. We use standard deviation (SD) to measure the imbalance in resource allocation, which is defined as $\sqrt{(1 /|E|) \cdot \sum_{l}\left(\left(u_{l} / W\right)-(1 /|E|) \sum_{l}\left(u_{l} / W\right)\right)^{2}}, l \in E$. The AVG and SD for transmitter utilization, receiver utilization, and electronic interface utilization are similarly defined.

On average, the SASR schemes use less network resources under both light and heavy traffic loads, simply because SPF routing consumes less network resources. DASR has both highest AVG values and highest SD values. This suggests that its performance improvement over the SASR schemes in terms of blocking probability only relies on its capability of sharing resources between access and regeneration functions. Although having more available regeneration resources due to the sharing, the DASR scheme is neither capable of minimizing the number of regenerations nor capable of handling the imbalance in resource allocation, thus resulting in worse performance than the DADR-IDDBR scheme. The very low SD values of the DADR-IDDBR scheme under all traffic loads clearly show its capability in balancing resource allocation. 
TABLE III

Resource Utilization in the ENtire Network Under Configuration-2 (AVG: Average Utilization in Percentage; SD: STANDARD DEVIATION In PERCENTAGe)

\begin{tabular}{c|c|c|c|c|c|c|c|c}
\hline \multirow{2}{*}{$\begin{array}{c}\text { Category of } \\
\text { Resources }\end{array}$} & $\begin{array}{c}\text { Link } \\
\text { Utilization }\end{array}$ & $\begin{array}{c}\text { Transmitter } \\
\text { Utilization }\end{array}$ & $\begin{array}{c}\text { Receiver } \\
\text { Utilization }\end{array}$ & $\begin{array}{c}\text { Electronic Interface } \\
\text { Utilization }\end{array}$ \\
\cline { 2 - 8 } & $A V G$ & $S D$ & $A V G$ & $S D$ & $A V G$ & $S D$ & $A V G$ & $S D$ \\
\hline \hline \multicolumn{8}{c}{ Configuration-2 with 40 established local routes in four domains } \\
\hline SASR-1/4 & $8.8 \%$ & $2.9 \%$ & $11.4 \%$ & $4.0 \%$ & $12.3 \%$ & $4.0 \%$ & $12.3 \%$ & $3.7 \%$ \\
\hline SASR-1/2 & $9.4 \%$ & $3.5 \%$ & $12.4 \%$ & $5.2 \%$ & $12.6 \%$ & $4.6 \%$ & $12.7 \%$ & $4.2 \%$ \\
\hline DASR & $10.1 \%$ & $4.2 \%$ & $13.5 \%$ & $5.2 \%$ & $13.5 \%$ & $5.0 \%$ & $13.5 \%$ & $5.0 \%$ \\
\hline DADR-IDDBR & $10.0 \%$ & $3.1 \%$ & $13.2 \%$ & $4.1 \%$ & $13.0 \%$ & $3.3 \%$ & $13.1 \%$ & $2.9 \%$ \\
\hline \hline \multicolumn{8}{|c|}{ Configuration-2 with 160 established local routes in four domains } \\
\hline SASR-1/4 & $34.9 \%$ & $11.1 \%$ & $42.3 \%$ & $11.4 \%$ & $43.6 \%$ & $12.0 \%$ & $42.9 \%$ & $10.6 \%$ \\
\hline SASR-1/2 & $35.9 \%$ & $12.8 \%$ & $46.0 \%$ & $12.1 \%$ & $45.5 \%$ & $13.2 \%$ & $47.2 \%$ & $11.7 \%$ \\
\hline DASR & $38.4 \%$ & $14.5 \%$ & $49.2 \%$ & $11.3 \%$ & $48.3 \%$ & $12.9 \%$ & $49.5 \%$ & $12.3 \%$ \\
\hline DADR-IDDBR & $37.3 \%$ & $11.0 \%$ & $50.1 \%$ & $7.0 \%$ & $48.9 \%$ & $9.3 \%$ & $49.1 \%$ & $6.5 \%$ \\
\hline
\end{tabular}

TABLE IV

RESOURCE UtilizATION AT INTERIOR REgENERATION CAPABLE NODES AND AT BOUNDARY NODES UNDER CONFIGURATION-4 (TU: TRANSMITTER UTILIZATION; RU: RECEIVER UTILIZATION; EU: ELECTRONIC INTERFACE UTILIZATION)

\begin{tabular}{|c|c|c|c|c|c|c|c|c|c|c|c|c|}
\hline \multirow{3}{*}{$\begin{array}{l}\text { Category of } \\
\text { Resources }\end{array}$} & \multicolumn{6}{|c|}{ At Interior Regeneration Capable Nodes } & \multicolumn{6}{|c|}{ At Boundary Nodes } \\
\hline & \multicolumn{2}{|c|}{ TU } & \multicolumn{2}{|c|}{ RU } & \multicolumn{2}{|c|}{$\mathbf{E U}$} & \multicolumn{2}{|c|}{ TU } & \multicolumn{2}{|c|}{$\mathbf{R U}$} & \multicolumn{2}{|c|}{$\mathbf{E U}$} \\
\hline & $A V G$ & $S D$ & $A V G$ & $S D$ & $A V G$ & $S D$ & $A V G$ & $S D$ & $A V G$ & $S D$ & $A V G$ & $S D$ \\
\hline \multicolumn{13}{|c|}{ Configuration- 4 with 40 established local routes in four domains } \\
\hline SASR- $1 / 4$ & $10.1 \%$ & $3.5 \%$ & $11.3 \%$ & $3.6 \%$ & $11.2 \%$ & $3.6 \%$ & $6.3 \%$ & $1.5 \%$ & $6.7 \%$ & $1.7 \%$ & $6.9 \%$ & $1.6 \%$ \\
\hline SASR-1/2 & $12.7 \%$ & $4.4 \%$ & $11.9 \%$ & $4.5 \%$ & $13.1 \%$ & $4.6 \%$ & $6.7 \%$ & $2.2 \%$ & $7.0 \%$ & $1.5 \%$ & $7.1 \%$ & $2.0 \%$ \\
\hline DASR & $12.9 \%$ & $4.6 \%$ & $13.2 \%$ & $5.2 \%$ & $13.4 \%$ & $4.5 \%$ & $7.9 \%$ & $2.7 \%$ & $7.0 \%$ & $2.5 \%$ & $8.2 \%$ & $2.4 \%$ \\
\hline DADR-IDDBR & $9.8 \%$ & $3.0 \%$ & $10.9 \%$ & $2.7 \%$ & $10.2 \%$ & $2.7 \%$ & $11.3 \%$ & $2.9 \%$ & $9.3 \%$ & $2.6 \%$ & $11.5 \%$ & $2.8 \%$ \\
\hline \multicolumn{13}{|c|}{ Configuration-4 with 160 established local routes in four domains } \\
\hline SASR- $1 / 4$ & $44.3 \%$ & $13.9 \%$ & $42.2 \%$ & $13.1 \%$ & $43.2 \%$ & $12.4 \%$ & $32.5 \%$ & $7.3 \%$ & $30.0 \%$ & $7.0 \%$ & $30.5 \%$ & $6.2 \%$ \\
\hline SASR- $1 / 2$ & $45.0 \%$ & $14.2 \%$ & $44.3 \%$ & $15.9 \%$ & $44.8 \%$ & $13.1 \%$ & $35.1 \%$ & $7.2 \%$ & $33.3 \%$ & $6.8 \%$ & $33.1 \%$ & $6.9 \%$ \\
\hline DASR & $45.1 \%$ & $16.0 \%$ & $48.0 \%$ & $16.2 \%$ & $46.7 \%$ & $11.4 \%$ & $33.2 \%$ & $6.4 \%$ & $34.6 \%$ & $6.8 \%$ & $34.0 \%$ & $6.6 \%$ \\
\hline DADR-IDDBR & $39.7 \%$ & $12.3 \%$ & $37.5 \%$ & $10.5 \%$ & $39.1 \%$ & $9.2 \%$ & $41.6 \%$ & $6.0 \%$ & $40.2 \%$ & $5.4 \%$ & $40.7 \%$ & $5.4 \%$ \\
\hline
\end{tabular}

TABLE $\mathrm{V}$

RUNNING TIMES (PC: LINUX+1.2 GHz AMD ATHLON PROCESSOR)

\begin{tabular}{l|c|c|c|c|c}
\hline SASR-1/2 and SASR-1 $1 / 4$ & \multicolumn{2}{c|}{ DASR } & \multicolumn{2}{c}{ DADR-IDDBR } \\
\hline \hline Average & Maximum & Average & Maximum & Average & Maximum \\
\hline $2.4 \mathrm{~ms}$ & $4.2 \mathrm{~ms}$ & $3.6 \mathrm{~ms}$ & $6.5 \mathrm{~ms}$ & $54.0 \mathrm{~ms}$ & $239.4 \mathrm{~ms}$ \\
\hline
\end{tabular}

In Table IV, we further divide the statistics between the interior regeneration capable nodes and the boundary nodes. Under Configuration-4, a boundary node has twice as many transmitters, receivers, and electronic interfaces as an interior regeneration capable node. For the SASR and DASR schemes, the higher resource utilization at the interior regeneration capable nodes than at the boundary nodes suggests that both schemes perform badly in handling such an imbalance. This is also one of the reasons that they are outperformed by DADR-IDDBR in terms of blocking probability. For each arriving local route request, DADR-IDDBR can dynamically carry out regeneration at the nodes that have more regeneration resources and therefore can balance resource utilization between the boundary nodes and the interior regeneration capable nodes.

\section{Running Times}

Performance improvement of the DADR-IDDBR scheme over other schemes does not come without cost. Table V shows that the SASR schemes run fastest, followed by the DASR scheme. All these schemes also have quite uniform running times for all route requests. DADR-IDDBR is more than ten times slower than other schemes. We ascribe this time cost to the complex OC-BFS search procedure and its iterative invocation in the IDDBR algorithm. Due to the variation in the number of OC-BFS invocations, the maximum DADR-IDDBR running time is up to four times longer than its average running time.

The DADR-IDDBR algorithm is designed for dynamic routing in large-sized WDM networks. Such a network can be up to ten times larger than the example network studied in this paper and may use a larger number (more than 16) of wavelengths on each link. According to the time complexity expression in Section $\mathrm{V}-\mathrm{C}$, the average running time of DADR-IDDBR can increase to $10 \sim 20 \mathrm{~s}$ with a maximum of $50 \sim 100 \mathrm{~s}$. If a routing table is precalculated periodically, this time is acceptable since optical-layer link states do not change 
very frequently. For a routing-on-demand style provisioning, this time is quite long but may still be acceptable for some applications in consideration of the network size. Although designed as a dynamic routing algorithm, DADR-IDDBR is not appropriate for dynamic optical-layer lightpath restoration, which requires very fast computation.

\section{CONCLUSION}

In this paper, we addressed the problem of dynamic routing in translucent WDM optical networks. This paper departed from previous studies by allowing for sharing of transmitters, receivers, and electronic interfaces between access and regeneration functions. We proposed a dynamic resource allocation and dynamic routing scheme to boost efficiency in such a network. We developed a translucent dynamic routing algorithm (IDDBR) by integrating complex optical-layer constraints with an optical-layer route search procedure and a balanced regeneration resource allocation procedure. IDDBR is only an intradomain routing algorithm but it also enables resultant local routes to serve different roles in end-to-end lightpaths, and hence simplifies the design of interdomain routing schemes. Our experimental results show that the IDDBR-based dynamic resource allocation and dynamic routing scheme outperforms those schemes that use static resource allocation and/or static routing in terms of blocking probability and resource utilization, albeit using a reasonably longer running time. An interdomain routing scheme that establishes end-to-end lightpaths crossing multiple domains can be realized based on the proposed IDDBR intradomain routing algorithm. For the interdomain case, we refer the interested readers to our study in [41].

\section{REFERENCES}

[1] R. Sabella, E. Iannone, M. Listanti, M. Berdusco, and S. Binetti, "Impact of transmission performance on path routing in all-optical transport networks," J. Lightw. Technol., vol. 16, no. 11, pp. 1965-1972, Nov. 1998.

[2] C. A. Brackett, A. S. Acampora, J. Sweitzer, G. Tangonan, M. T. Smith, W. Lennon, K. C. Wang, and R. H. Hobbs, "A scalable multiwavelength multihop optical network: A proposal for research on all-optical networks," J. Lightw. Technol., vol. 11, no. 5, pp. 736-753, May-Jun. 1993.

[3] B. Ramamurthy, H. Feng, D. Datta, J. P. Heritage, and B. Mukherjee, "Transparent vs. opaque vs. translucent wavelength-routed optical networks," in Optical Fiber Communications Conf. (OFC) 1999 Tech. Dig., vol. 1, San Diego, CA, Feb. 1999, pp. 59-61.

[4] B. Ramamurthy, S. Yaragorla, and X. Yang, "Translucent optical WDM networks for the next-generation backbone networks," in Proc. IEEE GLOBECOM, vol. 1, San Antonio, TX, Nov. 2001, pp. 60-64.

[5] X. Yang and B. Ramamurthy, "Sparse regeneration in a translucent WDM optical network," in Proc. SPIE Asia-Pacific Optical Wireless Communications, Beijing, China, Nov. 2001, pp. 61-70.

[6] A. Saleh, "Islands of transparency - an emerging reality in multiwavelength optical networking," presented at the IEEE/LEOS Summer Topical Meeting Broadband Optical Networks Technologies, Monterey, CA, Jul. 1998.

[7] E. Mannie, Ed., (2003, Mar.) "Generalized multi-protocol label switching (GMPLS) architecture,'Internet Draft, Work in Progress. [Online]. Available: draft-ietf-ccamp-gmpls-architecture-05.txt

[8] R. E. Wagner, L. Nederlof, and S. De Maesschalck, "The potential of optical layer networks," in Optical Fiber Communications Conf. (OFC) 2001 Tech. Dig., Anaheim, CA, Mar. 2001, pp. TuT3.1-TuT3.3.

[9] R. E. Wagner, "Managed reach optical networks," in LEOS Summer Topical Meetings-2000 Dig., FL, Jul. 2000, pp. IV33-IV34.
[10] T. Carpenter, D. Shallcross, J. Gannett, J. Jackel, and V. Von Lehmen, "Maximizing the transparency advantage in optical networks," in $O p$ tical Fiber Communications Conf. (OFC) 2003 Tech. Dig., vol. 2, Atlanta, GA, Mar. 2003, pp. 616-617.

[11] N. Antoniades, I. Roudas, R. E. Wagner, T. E. Stern, J. L. Jackel, and D. H. Richards, "Evaluating the reach of multiwavelength optical networks," in Proc. LEOS 1998 Annu. Meeting, vol. 1, Orlando, FL, Dec. 1998, pp. 284-285.

[12] H. Zang, J. P. Jue, and B. Mukherjee, "A review of routing and wavelength assignment approaches for wavelength-routed optical WDM networks," SPIE/Baltzer Optical Network Mag., vol. 1, no. 1, pp. 47-60, Jan. 2000

[13] K. Kompella and Y. Rekhter, Eds., (2002, Dec.) "OSPF extensions in support of generalized MPLS," Internet Draft, Work in Progress. [Online]. Available: draft-ietf-ccamp-ospf-gmpls-extensions-09.txt

[14] Y. Huang, A. Gencata, J. P. Heritage, and B. Mukherjee, "Routing and wavelength assignment with quality-of-signal constraints in WDM networks," presented at the Eur. Conf. Optical Communications (ECOC 2002), Copenhagen, Denmark, Sep. 2002.

[15] M. Kodialam and T. B. Lakshman, "Integrated dynamic IP and wavelength routing in IP over WDM networks," in Proc. IEEE INFOCOM, vol. 1, Anchorage, AK, Apr. 2001, pp. 358-366.

[16] B. Rajagopalan, J. Luciani, and D. Awduche. (2003, Apr.) "IP over optical networks: A framework," Internet Draft, Work in Progress. [Online]. Available: draft-ietf-ipo-framework-04.txt

[17] Generalized MPLS signaling RSVP-TE extensions, in IETF RFC 3473, NWG, L. Berger, Ed., Jan. 2003.

[18] Generalized MPLS signaling CR-LDP extensions, in IETF RFC 3472, NWG, P. Ashwood-Smith and L. Berger, Eds., Jan. 2003.

[19] A. Banerjee, L. Drake, L. Lang, B. Turner, D. Awduche, L. Berger, K. Kompella, and Y. Rekhter, "Generalized multiprotocol label switching: An overview of routing and management enhancement," IEEE Commun. Mag., vol. 39, pp. 144-150, Jan. 2001.

[20] J. Lang, Ed., (2003, Mar.) "Link management protocol," Internet Draft, Work in Progress. [Online]. Available: draft-ietf-ccamp-lmp-08.txt

[21] J. Lang and D. Papadimitriou. (2003, Mar.) "SONET/SDH encoding for link management protocol (LMP) test messages," Internet Draft, Work in Progress. [Online]. Available: draft-ietf-ccamp-lmp-test-sonetsdh-02.txt

[22] A. Fredette and J. Lang, Eds., (2003, Mar.) "Link management protocol (LMP) for DWDM optical line systems," Internet Draft, Work in Progress. [Online]. Available: draft-ietf-ccamp-lmp-wdm-02.txt

[23] J. Strand and A. Chiu. (2002, Dec.) "Impairments and other constraints on optical layer routing," Internet Draft, Work in Progress. [Online]. Available: draft-ietf-ipo-impairments-04.txt

[24] K. Kompella and Y. Rekhter, Eds., (2002, Aug.) "Routing extensions in support of generalized MPLS," Internet Draft, Work in Progress. [Online]. Available: draft-ietf-ccamp-gmpls-routing-05.txt

[25] Network node interface for the optical transport network (OTN), ITU-T Rec. G.709, Feb. 2001.

[26] Generalized MPLS signaling functional description, in IETF RFC 3471, NWG, L. Berger, Ed., Jan. 2003.

[27] K. Kompella and Y. Rekhter. (2001, Sep.) "LSP hierarchy with MPLS TE," Internet Draft, Work in Progress. [Online]. Available: draft-ietfmpls-lsp-hierarchy-08.txt

[28] D. Katz, D. Yeung, and K. Kompella. (2002, Oct.) "Traffic engineering extensions to OSPF version 2," Internet Draft, Work in Progress. [Online]. Available: draft-katz-yeung-ospf-traffic-09.txt

[29] H. Smit and T. Li. (2002, Dec.) "IS-IS extensions for traffic engineering," Internet Draft, Work in Progress. [Online]. Available: draft-ietf-isis-traffic-04.txt

[30] Y. Xu, A. Basu, and Y. Xue. (2002, Jun.) “A BGP/GMPLS solution for inter-domain optical networking," Internet Draft, Work in Progress. [Online]. Available: draft-xu-bgp-gmpls-02.txt

[31] J. Strand, A. L. Chiu, and R. Tkach, "Issues for routing in the optical layer," IEEE Commun. Mag., vol. 39, no. 2, pp. 81-87, Feb. 2001.

[32] E. Desurvire, Erbium-Doped Fiber Amplifiers, Principles and Applications. New York: Wiley, 1994.

[33] B. Ramamurthy, D. Datta, H. Feng, J. P. Heritage, and B. Mukherjee, "Impact of transmission impairments on the teletraffic performance of wavelength-routed optical networks," J. Lightw. Technol., vol. 17, no. 10, pp. 1713-1723, Oct. 1999.

[34] H. Takahashi, K. Oda, and H. Toba, "Impact of crosstalk in an arrayedwaveguide multiplexer on $N * N$ optical interconnection," J. Lightw. Technol., vol. 14, no. 6, pp. 1097-1105, Jun. 1996. 
[35] J. Y. Zhou, R. Cadeddu, E. Casaccia, C. Cavazzoni, and M. J. O’Mahony, "Crosstalk in multiwavelength optical cross-connect networks," $J$. Lightw. Technol., vol. 14, no. 6, pp. 1423-1435, Jun. 1996.

[36] J. van Leeuwen, "Graph algorithms," in Handbook of Theoretical Computer Science, Volume A: Algorithms and Complexity, J. van Leeuwen, Ed. Cambridge, MA: MIT Press, 1990, pp. 525-632.

[37] (2002, Nov.) Network map. Global Crossing, Inc.. [Online]. Available: http://www.globalcrossing.com/xml/network/net_map.xml

[38] B. Ramamurthy, D. Datta, H. Feng, J. P. Heritage, and B. Mukherjee, "SIMON: A simulator for optical networks," in Proc. SPIE 5th Annu. Conf. All-Optical Networking, Boston, MA, Sep. 1999, pp. 130-135.

[39] L. Kazovasky, S. Benedetto, and A. Willner, Optical Fiber Communication Systems. Norwood, MA: Artech House, 1996, pp. 37-41.

[40] R. Ramaswami and K. N. Sivarajan, Optical Networks: A Practical Perspective. San Francisco, CA: Morgan Kaufmann, 1998, pp. 227-230.

[41] X. Yang and B. Ramamurthy, "Inter-domain wavelength routing in the next-generation translucent optical internet backbones," OSA J. Opt. Networking, vol. 3, no. 3, Mar. 2004.

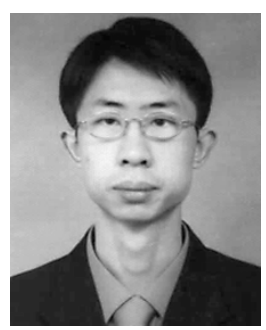

Xi Yang (S'03-M'05) received the B.S. degree in communication engineering the M.S. degree in communication and information systems from the University of Electronic Science and Technology, China, in 1997 and 2000, respectively, and the Ph.D. degree in computer science from the University of NebraskaLincoln in 2004.

He was with Lucent Technologies for one year as a Member of Technical Staff at Bell Laboratories, Beijing, China. He is currently a Research Associate with the University of Southern California Information Science Institute (USC/ISI). His research interests include design of WDM optical networks, wavelength routing and switching, optical network survivability, and generic network design, control, and management.

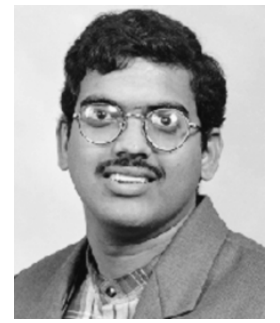

Byrav Ramamurthy (S'97-A'98-M'03) received the B.Tech. degree in computer science and engineering from Indian Institute of Technology, Madras, in 1993. He received the M.S. and Ph.D. degrees in computer science from the University of California (UC) Davis in 1995 and 1998, respectively.

Since August 1998, he has been an Assistant Professor in the Department of Computer Science and Engineering, University of Nebraska-Lincoln (UNL). He is the founding Codirector of the Advanced Networking and Distributed Experimental Systems (ANDES) Laboratory at UNL. He is the Feature Editor on Theses for Optical Network Magazine. He is author of Design of Optical WDM Networks—LAN, MAN and WAN Architectures (Norwood, MA: Kluwer Academic, 2000). His research areas include optical networks, distributed systems, and telecommunications.

Prof. Ramamurthy received the Indian National Talent Search scholarship and was a Fellow of the Professors for the Future program at UC Davis. He received the UNL Research Council Grant-in-Aid award for 1999 and the College of Engineering and Technology Faculty Research Award for 2000. He was a Guest Coeditor for a special issue of IEEE NETWORK magazine on Optical Communication Networks. He was a member of the Technical Program Committees for the IEEE INFOCOM, IEEE GLOBECOM, Opticomm, ICC, and ICCCN conferences. He was Secretary of the IEEE ComSoc Optical Networking Technical Committee (ONTC). 